16 March 2004

\title{
GENERALISED SHOT NOISE COX PROCESSES
}

JESPER MØLLER, ${ }^{*}$ Aalborg University

GIOVANNI LUCA TORRISI, ${ }^{* *}$ CNR-Istituto per le Applicazioni del Calcolo "M. Picone"

\begin{abstract}
We introduce a new class of Cox cluster processes called generalised shotnoise Cox processes (GSNCPs), which extends the definition of shot noise Cox processes (SNCPs) in two directions: the point process which drives the shot noise is not necessarily Poisson, and the kernel of the shot noise can be random. Thereby a very large class of models for aggregated or clustered point patterns is obtained. Due to the structure of GSNCPs, a number of useful results can be established. We focus first on deriving summary statistics for GSNCPs and next on how to make simulation for GSNCPs. Particularly, results for first and second order moment measures, reduced Palm distributions, the $J$-function, simulation with or without edge effects, and conditional simulation of the intensity function driving a GSNCP are given. Our results are exemplified for special important cases of GSNCPs, and we discuss the relation to corresponding results for SNCPs.

Keywords: Cluster process; conditional simulation; Cox process; edge effect; generalised shot noise G Cox process; geometric ergodicity; Markov point process; Metropolis-Hastings algorithm; Palm distribution; perfect simulation; shot noise Cox process; spatial point process; summary statistics
\end{abstract}

AMS 2000 Subject Classification: Primary 60D05;60G55;62M30

Secondary 60K35;68U20

\footnotetext{
* Postal address: Department of Mathematical Sciences, Aalborg University Fredrik Bajers Vej 7G, DK-9220 Aalborg, Denmark. Email address: jm@math.auc.dk

** Postal address: CNR-Istituto per le Applicazioni del Calcolo "M. Picone", Viale del Policlinico 137, I-00161 Rome, Italy. Email address: torrisi@iac.rm.cnr.it
} 


\section{Introduction}

One of the most important and versatile class of point process models for clustered point patterns is the class of Cox process models, see e.g. [8, 9, 11, 42]. Recently, simulation-based inference for certain families of Cox processes has been studied in great detail $[4,5,7,22,30,31,32,33,46]$. As explained in Section 2 this research has shown the need for extending existing model classes for Cox processes, and the present paper is therefore concerned with a new rich class of Cox process models, which to some extend is tractable for mathematical analysis and particularly for simulationbased inference.

Recall that a point process $X$ on $\mathbb{R}^{d}$ is a Cox process driven by a random field $Z(\xi) \geq 0, \xi \in \mathbb{R}^{d}$, if $X \mid Z$ is a Poisson process on $\mathbb{R}^{d}$ with intensity function $Z$. Throughout this paper $X$ denotes a Cox process driven by a random field of the form

$$
Z(\xi)=\sum_{j} \gamma_{j} k_{b_{j}}\left(c_{j}, \xi\right)
$$

where

- the $\left(c_{j}, b_{j}, \gamma_{j}\right)$ are the points of a point process $\Phi$ on $\mathbb{R}^{d} \times(0, \infty) \times(0, \infty)$,

- we identify $\sum_{j}$ and $\sum_{\left(c_{j}, b j, \gamma_{j}\right) \in \Phi}$,

- and $b_{j}>0$ is a bandwidth for the kernel $k_{b_{j}}\left(c_{j}, \cdot\right)$ :

$$
k_{b_{j}}\left(c_{j}, \xi\right)=k_{1}\left(c_{j} / b_{j}, \xi / b_{j}\right) / b_{j}^{d}
$$

where $k_{1}\left(c_{j}, \cdot\right)$ is a density with respect to Lebesgue measure on $\mathbb{R}^{d}$.

We call $X$ a generalised shot noise Cox process (GSNCP), since a shot noise Cox process $(S N C P)$ is the special case with $\left\{\left(c_{j}, \gamma_{j}\right)\right\}$ a Poisson process on $\mathbb{R}^{d} \times(0, \infty)$ and all $b_{j}$ equal and fixed, cf. Møller [29] (extending previous work by Brix [3] and Wolpert and Ickstadt [46]). We can also view a GSNCP as a Cox cluster process, since $X \mid \Phi$ is distributed as the superposition $\bigcup_{j} X_{j}$ of independent Poisson processes $X_{j}$ with intensity functions $\gamma_{j} k_{b_{j}}\left(c_{j}, \cdot\right)$. In applications of $(2)$ the kernel $k_{1}\left(c_{j}, \cdot\right)$ is usually concentrated around $c_{j}$, so we call $\Phi_{\text {cent }}=\left\{c_{j}\right\}$ the centre (or mother) process and $X_{j}$ the cluster (or daughter process) with centre (or mother) $c_{j}$, intensity $\gamma_{j}$, and dispersion density $k_{b_{j}}\left(c_{j}, \cdot\right)$. Many of the results in this paper can easily be modified to 
hold for a general stochastic kernel $k_{b_{j}}\left(c_{j}, \cdot\right)$ where $b_{j}$ is a random variable with state space given by an arbitrary metric space $E$, but for specificity and ease of presentation we let $E=(0, \infty)$ and interpret $b_{j}$ as a bandwidth.

The focus in this paper is on the probabilistic aspects of GSNCPs, with a view to statistical applications. Although GSNCPs are more complicated than SNCPs, we show that due to the structure of GSNCPs, still a number of useful and general results can be established. Our results are exemplified for special important cases of GSNCPs, and we discuss the relation to corresponding results for SNCPs.

The paper is organised as follows. Section 2 motivates the introduction of GSNCPs and contains some other preliminaries, including examples of GSNCPs. Section 3 concerns results for summary statistics and reduced Palm distributions of GSNCPs. Particularly, results for first and second order properties and the $J$-function are obtained. Section 4 deals with algorithms for simulation of a GSNCP X within a bounded window $W$, and for conditional simulation of $\Phi$ given the restriction of $X$ to $W$. We quantify the effect of ignoring edge effects in a straightforward simulation algorithm for $X$ when e.g. $\Phi_{\text {cent }}$ is restricted to a bounded extended window $W_{\text {ext }} \supset W$. We also describe a perfect simulation algorithm inspired by the work of Brix and Kendall [6]. Further, for a certain Metropolis-Hastings algorithm for conditional simulation of $\Phi$ given $X \cap W$, we discuss convergence properties, particularly we establish geometric ergodicity. Finally, Section 5 contains a brief discussion of future research and the importance of our results for statistical inference.

\section{Background}

\subsection{Motivation}

Our extension of SNCPs to GSNCPs is motivated by different statistical applications:

(a) For Neyman-Scott processes (see Example 2 in Section 2.3) and many other Cox cluster processes used for statistical analysis (see e.g. [11, 33, 42]), $\Phi_{\text {cent }}$ is assumed to be a Poisson process and the $\left(b_{j}, \gamma_{j}\right)$ are assumed to be equal to an unknown parameter. As illustrated by van Lieshout and Baddeley [22], a repulsive Markov point process model for $\Phi_{\text {cent }}$ may be more relevant in many 
situations. Such models may be described by GSNCPs but not by SNCPs.

(b) Wolpert and Ickstadt [46] considered a fully Bayesian analysis for a Poissongamma model (a particular case of an SNCP, see Example 3 in Section 2.3): a prior for the parameter in a parametric model for the intensity function of the Poisson process $\Phi$ is imposed, whereby $X$ becomes a GSNCP.

(c) Møller and Waagepetersen [33] considered likelihood inference for parametric models of SNCPs, where the likelihood is in general not available in closed form and the underlying random field $Z$ is unobserved. For different fixed values of the bandwidths, they combined Markov chain Monte Carlo (MCMC) methods for calculating maximum partial likelihood estimates and likelihood ratios, whereby an approximate MLE was obtained (see Section 10.3 in [33]). This procedure is rather time consuming, and it would be easier to use a Bayesian MCMC approach, imposing a prior on the bandwidth. Then a GSNCP model (with all $b_{j}$ equal but random) is obtained for the likelihood term in the posterior density.

(d) SNCPs are often claimed to allow a certain degree of flexibility [28, 31, 38, 46]. In an SNCP, for the random intensity function $Z$ in (1), the random cluster intensity $\gamma_{j}$ is scaling the kernel $k_{b}\left(c_{j}, \cdot\right)$ where $b>0$ is the fixed bandwidth. However, the same degree of flexibility for modelling $Z$ may be obtained by letting the $\gamma_{j}$ be equal but using different random bandwidths $b_{j}$. Even more flexibility is of course obtained when both the $\gamma_{j}$ and the $b_{j}$ are random as in a GSNCP.

(e) Kingman [19] (see also Section 5.5 in [11]) considered a model for reproducing individuals, where the $(n+1)$ th generation $G_{n+1}$ given the $n$th generation $G_{n}$ (and previous generations $G_{0}, \ldots, G_{n-1}$ ) is a Poisson process with an intensity measure of the form (1), where the cluster centres are given by $G_{n}$, each $\gamma_{j}$ is a function of $\left(c_{j}, G_{n}\right)$, and the bandwidths are equal and fixed. Thus $G_{n+1}$ becomes a GSNCP but not necessarily an SNCP. Furthermore, assuming that $G_{0}$ is a Poisson process, the superposition of GSNCPs $G_{0}, G_{1}, \ldots$, can be interpreted as a spatial Hawkes process (extending the definition in $[15,16]$ for $d=1$ to $d \geq 1$ and allowing a more general structure of the conditional intensity function). 
We consider in particular the following models for $\Phi$. Cox process models for $\Phi$ appear naturally when we consider a hierarchical model where $\Phi \mid \theta$ is a Poisson process with an intensity measure $\zeta_{\theta}$ depending on a random variable $\theta$; see points (b) and (d) above and Example 3 in Section 2.3. The case where $\Phi_{\text {cent }}$ and $\left\{\left(b_{j}, \gamma_{j}\right)\right\}$ are independent is particularly tractable, and in our opinion the most important models for $\Phi_{\text {cent }}$ are then Poisson models and Markov point process models; see point (a) above and Example 4 in Section 2.3. Markov point process models provide flexible models for inhibition between the cluster centres, while Poisson models provide a mathematical convenient framework, cf. [29, 33].

\subsection{Assumptions and other preliminaries}

This section specifies certain conditions which are assumed throughout the text. Also some terminology and notation are introduced.

We assume that $\Phi$ is a random locally finite subset of $\Omega=\mathbb{R}^{d} \times(0, \infty) \times(0, \infty)$, i.e. $\Phi_{D} \equiv \Phi \cap D$ is finite whenever $D \subset \Omega$ is bounded. Moreover, $Z$ is assumed to be almost surely locally integrable, i.e. with probability $1, \int_{B} Z(\xi) \mathrm{d} \xi<\infty$ for bounded Borel sets $B \subset \mathbb{R}^{d}$. Hence, for any bounded $B \subset \mathbb{R}^{d}, X_{B} \equiv X \cap B$ is almost surely finite, and therefore only finitely many clusters $X_{j}$ have points in $B$. Note that the centre process $\Phi_{\text {cent }}$ is countable, but not necessarily locally finite (see Example 3 in Section 2.3).

If $\Phi_{\text {cent }}$ is stationary (i.e. its distribution is invariant under translations in $\mathbb{R}^{d}$ ) and $k_{1}(c, \xi)=k_{1}(\xi-c)$ is invariant under translations in $\mathbb{R}^{d}$, then $Z$ and hence $X$ is stationary. If furthermore the distribution of $\Phi_{\text {cent }}$ is invariant under motions in $\mathbb{R}^{d}$ and $k_{1}$ is isotropic, i.e. $k_{1}(c, \xi)$ depends only on the distance $\|c-\xi\|$, then the distributions of $Z$ and $X$ are invariant under motions in $\mathbb{R}^{d}$. Two frequently used choices of isotropic kernels are the Gaussian kernel

$$
k_{1}(\xi)=(2 \pi)^{-d / 2} \exp \left(-\|\xi\|^{2} / 2\right)
$$

and the uniform kernel

$$
k_{1}(\xi)=\mathbf{1}[\|\xi\| \leq 1] / \omega_{d}
$$

where $\mathbf{1}[\cdot]$ denotes the indicator function and $\omega_{d}=\pi^{d / 2} / \Gamma(1+d / 2)$.

We shall often refer to the intensity measure and the second order reduced moment 
measure of $\Phi$, which we denote by $\zeta$ and $\zeta^{(2)}$, respectively. Recall that for measurable functions $h_{1}: \Omega \mapsto[0, \infty)$ and $h_{2}: \Omega \times \Omega \mapsto[0, \infty)$,

$$
\int h_{1}(c, b, \gamma) \mathrm{d} \zeta(c, b, \gamma)=\mathrm{E} \sum_{j} h_{1}\left(c_{j}, b_{j}, \gamma_{j}\right)
$$

and

$$
\int h_{2}\left((c, b, \gamma),\left(c^{\prime}, b^{\prime}, \gamma^{\prime}\right)\right) \mathrm{d} \zeta^{(2)}\left((c, b, \gamma),\left(c^{\prime}, b^{\prime}, \gamma^{\prime}\right)\right)=\mathrm{E} \sum_{j \neq j^{\prime}} h_{2}\left(\left(c_{j}, b_{j}, \gamma_{j}\right),\left(c_{j^{\prime}}, b_{j^{\prime}}, \gamma_{j^{\prime}}\right)\right)
$$

see e.g. [42]. In applications, $\zeta$ is often of the form

$$
\zeta(D)=\iint_{D} \mathrm{~d} c \mathrm{~d} \chi(b, \gamma)
$$

for Borel sets $D \subseteq \Omega$, where $\chi$ is a measure on $(0, \infty) \times(0, \infty)$, cf. Examples $2-4$ in Section 2.3.

\subsection{Examples}

The following examples describe important model classes of GSNCPs. As the measures $\zeta$ and $\zeta^{(2)}$ introduced above play an important role in Section 3, we specify these measures in the examples.

Example 1. A particular tractable model class is obtained when $\Phi$ is a Poisson process with locally finite intensity measure $\zeta$. This class contains the SNCPs (the special case where all bandwidths are equal and fixed). Note that $\zeta^{(2)}=\zeta \times \zeta$ is just a product measure, and (7) is equivalent to stationarity of $\Phi_{\text {cent }}$.

Example 2. A Neyman-Scott process [35] is obtained when $\Phi_{\text {cent }}$ is a stationary Poisson process on $\mathbb{R}^{d}$ and the cluster intensities $\gamma_{j}=\gamma$ and the bandwidths $b_{j}=b$ are equal and fixed. For the Gaussian kernel (3) we have a (modified) Thomas process [44], and for the uniform kernel (4) we have a Matérn cluster process [24, 25].

Natural extensions of this model include GSNCPs obtained if $\Phi_{\text {cent }}$ is a stationary point process on $\mathbb{R}^{d}$ with intensity $\rho_{\text {cent }}<\infty, \Phi_{\text {cent }}$ is independent of the $\left(b_{j}, \gamma_{j}\right)$, and either

(i) the $\left(b_{j}, \gamma_{j}\right)=(b, \gamma)$ are identical with distribution $Q$, or 
(ii) the $b_{j}=b$ are identical with distribution $Q_{1}$, the $\gamma_{j}$ are i.i.d. with distribution $Q_{2}$, and $b$ and $\left\{\gamma_{j}\right\}$ are independent, or

(iii) the $\left(b_{j}, \gamma_{j}\right)$ are i.i.d. with distribution $Q$.

We call such extensions for generalised Neyman-Scott processes. Then (7) holds where

$$
\chi=\rho_{\text {cent }} Q
$$

with $Q=Q_{1} \times Q_{2}$ in case (ii). This follows by conditioning on $\Phi_{\text {cent }}$ in the right hand side of (5) and using standard arguments, where we first let $h_{1}(c, b, \gamma)$ in (5) be an indicator function $\mathbf{1}[c \in A, b \in B, \gamma \in C]$. Furthermore, using similar arguments and (6), we obtain

$$
\begin{aligned}
& \int h_{2}\left((c, b, \gamma),\left(c^{\prime}, b^{\prime}, \gamma^{\prime}\right)\right) \mathrm{d} \zeta^{(2)}\left((c, b, \gamma),\left(c^{\prime}, b^{\prime}, \gamma^{\prime}\right)\right) \\
& = \begin{cases}\iint h_{2}\left((c, b, \gamma),\left(c^{\prime}, b, \gamma\right)\right) \mathrm{d} Q(b, \gamma) \mathrm{d} \zeta_{\text {cent }}^{(2)}\left(c, c^{\prime}\right) & \text { in case (i) } \\
\iiint \int h_{2}\left((c, b, \gamma),\left(c^{\prime}, b, \gamma^{\prime}\right)\right) \mathrm{d} Q_{1}(b) \mathrm{d} Q_{2}(\gamma) \mathrm{d} Q_{2}\left(\gamma^{\prime}\right) \mathrm{d} \zeta_{\text {cent }}^{(2)}\left(c, c^{\prime}\right) & \text { in case (ii) } \\
\iiint h_{2}\left((c, b, \gamma),\left(c^{\prime}, b^{\prime}, \gamma^{\prime}\right)\right) \mathrm{d} Q(b, \gamma) \mathrm{d} Q\left(b^{\prime}, \gamma^{\prime}\right) \mathrm{d} \zeta_{\text {cent }}^{(2)}\left(c, c^{\prime}\right) & \text { in case (iii) }\end{cases}
\end{aligned}
$$

where $\zeta_{\text {cent }}^{(2)}$ denotes the second order reduced moment measure for $\Phi_{\text {cent }}$. Especially, if $\Phi_{\text {cent }}$ is a stationary Poisson process, then $\mathrm{d} \zeta_{\text {cent }}^{(2)}\left(c, c^{\prime}\right)=\rho_{\text {cent }}^{2} \mathrm{~d} c \mathrm{~d} c^{\prime}$ (see e.g. [42]).

Example 3. Suppose that $\left\{\left(c_{j}, \gamma_{j}\right)\right\}$ is a Poisson process on $\mathbb{R}^{d} \times(0, \infty)$ with intensity function

$$
\lambda_{\theta}(c, \gamma)=\lambda_{\theta}(\gamma)=\kappa \gamma^{-\alpha-1} \exp (-\tau \gamma) / \Gamma(1-\alpha)
$$

where $\theta=(\alpha, \kappa, \tau)$ is a parameter with $\alpha<1, \kappa>0$, and $\tau>0$ (these restrictions are equivalent to local integrability of $\lambda_{\theta}$ ). When all the $b_{j}$ in (1) are equal and fixed, $X$ is called a shot noise G Cox process (SNGCP) [3, 29]; a Poisson-gamma process [46] is the special case $\alpha=0$. In the stationary case, a SNGCP has intensity equal to $\kappa \tau^{\alpha-1}$.

We shall later refer to the following properties of the Poisson process $\left\{\left(c_{j}, \gamma_{j}\right)\right\}$. The two point processes $\left\{c_{j}\right\}$ and $\left\{\gamma_{j}\right\}$ are independent, and their distributions depend on the value of $\alpha$ as follows.

(i) $\quad \alpha<0$ : Then $\Phi_{\text {cent }}$ is a stationary Poisson process with intensity $-\kappa \tau^{\alpha} / \alpha$, and the $\gamma_{j}$ are independent and gamma distributed with shape parameter $-\alpha$ and inverse scale parameter $\tau$. 
(ii) $0 \leq \alpha<1$ : Then $\Phi_{\text {cent }}$ is not locally finite. However, $\left\{\left(c_{j}, \gamma_{j}\right): c_{j} \in A\right\}$ and $\left\{\left(c_{j}, \gamma_{j}\right): c_{j} \in B\right\}$ are independent for disjoint Borel sets $A, B \subset \mathbb{R}^{d}$. If $A$ has finite Lebesgue measure $|A|$, then the points in $\Phi_{\text {cent }} \cap A$ are independent and uniformly distributed on $A$, and the corresponding $\gamma_{j}$ form an inhomogeneous Poisson process on $(0, \infty)$ with intensity function $|A| \lambda_{\theta}(\gamma)$.

Generalised shot noise G Cox processes can naturally be obtained in different ways. For specificity, let $\pi$ be a distribution imposed on the parameter $\theta=(\alpha, \kappa, \tau)$, and let $\left\{\left(c_{j}, \gamma_{j}\right)\right\} \mid \theta$ be a Poisson process with intensity function $\lambda_{\theta}$, cf. [46]. Further, consider (1) with $\left\{b_{j}\right\}$ and $\left(\theta,\left\{\left(c_{j}, \gamma_{j}\right)\right\}\right)$ independent, and the $b_{j}$ either identical or i.i.d. with distribution $\nu$. Then we obtain a GSNCP where (7) holds with

$$
\chi(A)=\iint_{A} \operatorname{E} \lambda_{\theta}(\gamma) \mathrm{d} \nu(b) \mathrm{d} \gamma
$$

for Borel sets $A \subseteq(0, \infty) \times(0, \infty)$, where the expectation is with respect to $\pi$ (this follows by similar arguments as in Example 2). Furthermore,

$$
\begin{aligned}
& \int h_{2}\left((c, b, \gamma),\left(c^{\prime}, b^{\prime}, \gamma^{\prime}\right)\right) \mathrm{d} \zeta^{(2)}\left((c, b, \gamma),\left(c^{\prime}, b^{\prime}, \gamma^{\prime}\right)\right) \\
& =\iiint \iint h_{2}\left((c, b, \gamma),\left(c^{\prime}, b, \gamma^{\prime}\right)\right) \mathrm{E}\left(\lambda_{\theta}(\gamma) \lambda_{\theta}\left(\gamma^{\prime}\right)\right) \mathrm{d} c \mathrm{~d} c^{\prime} \mathrm{d} \nu(b) \mathrm{d} \gamma \mathrm{d} \gamma^{\prime}
\end{aligned}
$$

when the $b_{j}$ are identical, and

$$
\begin{aligned}
& \int h_{2}\left((c, b, \gamma),\left(c^{\prime}, b^{\prime}, \gamma^{\prime}\right)\right) \mathrm{d} \zeta^{(2)}\left((c, b, \gamma),\left(c^{\prime}, b^{\prime}, \gamma^{\prime}\right)\right) \\
& =\iiint \iiint h_{2}\left((c, b, \gamma),\left(c^{\prime}, b^{\prime}, \gamma^{\prime}\right)\right) \mathrm{E}\left(\lambda_{\theta}(\gamma) \lambda_{\theta}\left(\gamma^{\prime}\right)\right) \mathrm{d} c \mathrm{~d} c^{\prime} \mathrm{d} \nu(b) \mathrm{d} \nu\left(b^{\prime}\right) \mathrm{d} \gamma \mathrm{d} \gamma^{\prime}
\end{aligned}
$$

when the $b_{j}$ are i.i.d.

Example 4. As in van Lieshout and Baddeley [22] suppose that $\Phi_{\text {cent }}$ is a finite Markov (or Gibbs) point process [1, 20,33, 39, 40] defined on a bounded Borel set $B \subset \mathbb{R}^{d}$. This means that $\Phi_{\text {cent }}$ has a density $p$ with respect to the unit rate Poisson process on $B$ so that for finite subsets $\mathbf{c} \subset B$,

$$
p(\mathbf{c})=\prod_{y \subseteq \mathbf{c}} \varphi(y) .
$$

Here $\varphi(y) \geq 0$ is a so-called interaction function such that $\varphi(y)=1$ whenever $y$ contains two points larger than $R$ units apart, where $R<\infty$ is a parameter specifying 
the range of interaction. Note that if $p(\mathbf{c})>0$ and we define the so-called Papangelou conditional intensity by $\lambda^{*}(\mathbf{c}, \xi) \equiv p(\mathbf{c} \cup\{\xi\}) / p(\mathbf{c})$, then

$$
\lambda^{*}(\mathbf{c}, \xi)=\prod_{y \subseteq \mathbf{c} \cap b(\xi, R)} \varphi(y \cup\{\xi\})
$$

where $b(\xi, R)$ denotes the ball in $\mathbb{R}^{d}$ with centre $\xi$ and radius $R$.

In [22] it is also assumed that $Z(\xi)=\epsilon+\sum_{j} h\left(\xi \mid c_{j}\right)$ where $h\left(\xi \mid c_{j}\right)$ is a nonnegative integrable function and $\epsilon>0$ is a parameter. If instead $\epsilon=0$, we have a GSNCP with non-random $\left(\gamma_{j}, b_{j}\right)$, but clearly ( 7$)$ does not hold, since $\Phi_{\text {cent }}$ is contained in $B$. If moreover $\Phi_{\text {cent }}$ is independent of the $\left(b_{j}, \gamma_{j}\right)$, which are either i.i.d. or identical with distribution $Q$, then

$$
\int h_{1}(c, b, \gamma) \mathrm{d} \zeta(c, b, \gamma)=\iint h_{1}(\xi, b, \gamma) \mathrm{E} \lambda^{*}\left(\Phi_{\text {cent }}, \xi\right) \mathrm{d} \xi \mathrm{d} Q(b, \gamma) .
$$

This follows from the fact that $\Phi_{\text {cent }}$ has intensity function $\rho_{\text {cent }}(\xi)=\mathrm{E} \lambda^{*}\left(\Phi_{\text {cent }}, \xi\right)$ (see Proposition 6.2 in [33]), but a closed form expression for $\rho_{\text {cent }}$ is in general unknown (except in the Poisson case where $\lambda^{*}\left(\Phi_{\text {cent }}, \xi\right)$ does not depend on $\left.\Phi_{\text {cent }}\right)$. Furthermore,

$$
\begin{aligned}
& \int h_{2}\left((c, b, \gamma),\left(c^{\prime}, b^{\prime}, \gamma^{\prime}\right)\right) \mathrm{d} \zeta^{(2)}\left((c, b, \gamma),\left(c^{\prime}, b^{\prime}, \gamma^{\prime}\right)\right)=\iiint \int h_{2}\left((c, b, \gamma),\left(c^{\prime}, b, \gamma^{\prime}\right)\right) \\
& \mathrm{E}\left(\prod_{y \subseteq \Phi_{\text {cent }} \cap\left(b(c, R) \cup b\left(c^{\prime}, R\right)\right)} \varphi(y \cup\{c\}) \varphi\left(y \cup\left\{c^{\prime}\right\}\right) \varphi\left(y \cup\left\{c, c^{\prime}\right\}\right)\right) \mathrm{d} c \mathrm{~d} c^{\prime} \mathrm{d} Q(b, \gamma) \mathrm{d} Q\left(b^{\prime}, \gamma^{\prime}\right)
\end{aligned}
$$

see e.g. Proposition 6.2 in [33]. Also $\zeta^{(2)}$ is not known on closed form.

Suppose we instead consider a stationary Markov (or Gibbs) point process $\Phi_{\text {cent }}$ defined on $\mathbb{R}^{d}$, with an interaction function $\varphi$ of finite range of interaction $R$, where $\varphi$ is invariant under translations in $\mathbb{R}^{d}[12,33,36,37,41]$. Briefly, such a point process can be specified by a Papangelou conditional intensity $\lambda^{*}(\mathbf{c}, \xi)$ of the form (15) but now defined for locally finite subsets $\mathbf{c} \subset \mathbb{R}^{d}$ and points $\xi \in \mathbb{R}^{d} \backslash \mathbf{c}$ (for details, see the abovementioned references). If $\Phi_{\text {cent }}$ is independent of the $\left(b_{j}, \gamma_{j}\right)$, which are either i.i.d. or identical with distribution $Q$, then we have a GSNCP. Here (7) holds with $\chi$ of the form (8) provided

$$
\rho_{\text {cent }}=\mathrm{E} \lambda^{*}\left(\Phi_{\text {cent }}, 0\right)=\mathrm{E} \prod_{y \subseteq \Phi_{\text {cent }} \cap b(0, R)} \varphi(y \cup\{0\})
$$

is finite. However, a closed form expression for $\rho_{\text {cent }}$ is in general not known. Similarly for $\zeta^{(2)}$, which is still given by (17). 


\section{Summary statistics}

This section deals with summary statistics such as the intensity function, the pair correlation function, and the $J$-function for GSNCPs.

\subsection{First- and second-order characteristics}

Expressions for the product moments $\mathrm{E}\left[Z\left(\xi_{1}\right) \cdots Z\left(\xi_{n}\right)\right]$ in terms of the moment measures for $\Phi$ can be obtained in a similar way as in [29] by using the Slivnyak-Mecke theorem for the Poisson process $X \mid \Phi$. In this paper we concentrate on the two most fundamental summary statistics, namely the intensity function $\rho(\xi)=\mathrm{E} Z(\xi)$ and the pair correlation function $g(\xi, \eta)=\mathrm{E}[Z(\xi) Z(\eta)] /[\rho(\xi) \rho(\eta)]$ (provided that the means exist, and taking $\frac{0}{0}=0$ ). In the examples below we discuss to what extent closed form expressions for $\rho(\xi)$ and $g(\xi, \eta)$ can be derived for GSNCPs as introduced in Examples 1-4 in Section 2.3.

Proposition 1. The intensity function exists and is given by

$$
\rho(\xi)=\int \gamma k_{b}(c, \xi) \mathrm{d} \zeta(b, c, \gamma)
$$

provided that the integral is finite for all $\xi \in \mathbb{R}^{d}$.

Proof. Follows immediately from (5).

Example 5. By (19), if $(7)$ holds and $k_{1}(c, \xi)=k_{1}(\xi-c)$ is invariant under translations, $\rho=\rho(\xi)$ is given by

$$
\rho=\int \gamma \mathrm{d} \chi(b, \gamma)
$$

This reduces as follows for the GSNCPs in Examples 2 and 3: For a generalised Neyman-Scott process, $\rho=\rho_{\text {cent }} \mathrm{E} \gamma$, where the mean is with respect to $(b, \gamma) \sim Q$, cf. (8). For a generalised shot noise G Cox processes, $\rho=\mathrm{E}\left(\kappa \tau^{\alpha-1}\right)$, cf. (11).

However, for the Markov point process setting considered in Example 4, a closed form expression of $\rho(\xi)$ is in general unknown, since $\mathrm{E} \lambda^{*}\left(\Phi_{\text {cent }}, \xi\right)$ in $(16)$ or (18) is not known on closed form.

Proposition 2. The pair correlation function exists and is given by

$$
g(\xi, \eta)=\frac{\beta_{1}(\xi, \eta)+\beta_{2}(\xi, \eta)}{\rho(\xi) \rho(\eta)}
$$


provided that $\rho(\xi)<\infty$ for all $\xi \in \mathbb{R}^{d}$ and the integrals

$$
\beta_{1}(\xi, \eta)=\int \gamma \gamma^{\prime} k_{b}(c, \xi) k_{b^{\prime}}\left(c^{\prime}, \eta\right) \mathrm{d} \zeta^{(2)}\left((c, b, \gamma),\left(c^{\prime}, b^{\prime}, \gamma^{\prime}\right)\right)
$$

and

$$
\beta_{2}(\xi, \eta)=\int \gamma^{2} k_{b}(c, \xi) k_{b}(c, \eta) \mathrm{d} \zeta(c, b, \gamma)
$$

are finite for all $\xi, \eta \in \mathbb{R}^{d}$.

Proof. Follows along similar lines as in the proof of Proposition 1 in [29] but using (6).

Example 6. Suppose that $k_{1}(c, \xi)=k_{1}(\xi-c)$ is invariant under translations.

If $\Phi$ is a Poisson process so that $\Phi_{\text {cent }}$ is stationary and $\rho$ given by (20) is finite, then by Example 1 and Proposition 2,

$$
g(\xi, \eta)=1+\beta_{2}(\xi, \eta) / \rho^{2}
$$

where

$$
\beta_{2}(\xi, \eta)=\int \gamma^{2} \phi_{b}(\xi-\eta) \mathrm{d} \chi(b, \gamma)
$$

and

$$
\phi_{b}(\xi)=\int k_{b}(c) k_{b}(\xi+c) \mathrm{d} c .
$$

For the Gaussian kernel (3), this reduces to

$$
\phi_{b}(\xi)=\frac{1}{(4 \pi b)^{d / 2}} \exp \left(-\frac{\|\xi\|^{2}}{4 b^{2}}\right) .
$$

Consider a generalised Neyman-Scott process when $\Phi_{\text {cent }}$ is a stationary Poisson process with intensity $\rho_{\text {cent }}<\infty$. Then

$$
\beta_{2}(\xi, \eta)=\rho_{\text {cent }} \mathrm{E}\left(\gamma^{2} \phi_{b}(\xi-\eta)\right)
$$

where the mean is with respect to $(b, \gamma) \sim Q$. For each of the cases (i)-(iii) in Example 2 we obtain the following from (9) and Proposition 2. In the case (i), $\beta_{1}(\xi, \eta)=\rho_{\text {cent }}^{2} \mathrm{E}\left(\gamma^{2}\right)$, so

$$
g(\xi, \eta)=\frac{\mathrm{E}\left(\gamma^{2}\right)+\mathrm{E}\left(\gamma^{2} \phi_{b}(\xi-\eta)\right) / \rho_{\text {cent }}}{(\mathrm{E} \gamma)^{2}} .
$$


In the case (ii), $\beta_{1}(\xi, \eta)=\rho^{2}$, so

$$
g(\xi, \eta)=1+\frac{\mathrm{E}\left(\gamma^{2} \phi_{b}(\xi-\eta)\right)}{\rho_{\text {cent }}(\mathrm{E} \gamma)^{2}} .
$$

The case (iii) is just the special case of the Poisson case above with $\mathrm{d} \chi(b, \gamma)=$ $\rho_{\text {cent }} \mathrm{d} b \mathrm{~d} \gamma$, and $g$ is again of the form (22).

Consider next the specific example of a generalised shot noise $\mathrm{G}$ Cox process in Example 3. By (12) and (13), both when the $b_{j}$ are identical and when the $b_{j}$ are i.i.d., it is straightforwardly derived that $g$ is of the form (21) with

$$
\beta_{2}(\xi, \eta)=\mathrm{E}\left((1-\alpha) \kappa \tau^{\alpha-2}\right) \mathrm{E} \phi_{b}(\xi-\eta)
$$

where the means now are with respect to the independent random variables $b$ and $\theta=(\alpha, \kappa, \tau)$.

Note that $g \geq 1$ for the GSNCPs in Example 6. This is in accordance with the usual interpretation that $g \geq 1$ indicates aggregation of the points in $X[33,42]$. It seems to be an open problem to what extend $g \geq 1$ for the Markov point process setting considered in Example 4. Most Markov point process models are repulsive, that is, $\varphi(y) \leq 1$ whenever $\operatorname{card}(y) \geq 2$ (see e.g. [33]). In the special case of no interaction, i.e. $\varphi(y)=1$ whenever $\operatorname{card}(y) \geq 2$, we clearly have that $g=1$. So in the repulsive case, since aggregation in $X$ is expected to be more pronounced than if there is no interaction, one may conjecture that $g(\xi, \eta) \geq 1$, at least when $\xi$ and $\eta$ are sufficiently close. However, we have not succeeded in verify this by combining (16)-(18) and Proposition 2.

\subsection{Reduced Palm distributions and $J$-functions}

A simple description of the reduced Palm distribution of an SNCP was established in [29]. This section extends this to GSNCPs and discusses how to use this for deriving certain properties of van Lieshout and Baddeley's $J$-function [21].

We first need some additional notation and assumptions. Denote the state space of $X$ by $N_{\text {lf }}$, the set of locally finite subsets of $\mathbb{R}^{d}$. Let $N_{\text {lf }}$ be equipped with the $\sigma$-field $\mathcal{N}_{\text {lf }}$ generated by the sets $F_{B, n}=\left\{x \in N_{\text {lf }}: \operatorname{card}(x \cap B)=n\right\}$ for $n=0,1, \ldots$ and bounded Borel sets $B \subset \mathbb{R}^{d}$. Let the product space $N_{\mathrm{lf}} \times \mathbb{R}^{d}$ be equipped with the 
product $\sigma$-field $\mathcal{N}_{\text {lf }} \otimes \mathcal{B}^{d}$, where $\mathcal{B}^{d}$ is the Borel $\sigma$-field on $\mathbb{R}^{d}$. Similarly, for the point process $\Phi$ we define $\sigma$-fields by replacing $\mathbb{R}^{d}$ above with $\Omega=\mathbb{R}^{d} \times(0, \infty) \times(0, \infty)$.

Assume that the intensity function $\rho(\xi)$ for $X$ exists for Lebesgue almost all $\xi \in \mathbb{R}^{d}$, cf. Proposition 1. Recall that the reduced Palm distribution $P_{\xi}^{!}$of $X$ at $\xi \in \mathbb{R}^{d}$ and the reduced Palm distribution $P_{(c, b, \gamma)}^{!}$of $\Phi$ at $(c, b, \gamma) \in \mathbb{R}^{d} \times(0, \infty) \times(0, \infty)$ are given by the Campbell-Mecke formula: for Lebesgue almost all $\xi \in \mathbb{R}^{d}$ with $\rho(\xi)>0$ and for $\zeta$ almost all $(c, b, \gamma) \in \mathbb{R}^{d} \times(0, \infty) \times(0, \infty), P_{\xi}^{!}$and $P_{(c, b, \gamma)}^{!}$are uniquely given by

$$
\mathrm{E} \sum_{\xi \in X} f(X \backslash\{\xi\}, \xi)=\iint f(x, \xi) \rho(\xi) \mathrm{d} P_{\xi}^{!}(x) \mathrm{d} \xi
$$

for nonnegative measurable functions $f$ and

$$
\mathrm{E} \sum_{(c, b, \gamma) \in \Phi} h(\Phi \backslash\{(c, b, \gamma)\},(c, b, \gamma))=\iint h(\phi,(c, b, \gamma)) \mathrm{d} P_{(c, b, \gamma)}^{!}(\phi) \mathrm{d} \zeta(c, b, \gamma)
$$

for nonnegative measurable functions $h$; see $[9,42]$.

When $\rho(\xi)>0$ we define the following. Let

$$
Z_{\xi}(\eta)=\gamma_{\xi} k_{b_{\xi}}\left(c_{\xi}, \eta\right), \quad \eta \in \mathbb{R}^{d},
$$

where $\left(c_{\xi}, b_{\xi}, \gamma_{\xi}\right)$ is a random variable with distribution

$$
P\left(\left(c_{\xi}, b_{\xi}, \gamma_{\xi}\right) \in D\right)=\int_{D}\left[\gamma k_{b}(c, \xi) / \rho(\xi)\right] \mathrm{d} \zeta(c, b, \gamma)
$$

for Borel sets $D \subseteq \Omega$. Conditional on $\left(c_{\xi}, b_{\xi}, \gamma_{\xi}\right)$, let $X_{\xi}$ and $X^{\xi}$ denote independent point processes, where $X_{\xi}$ is a Poisson process on $\mathbb{R}^{d}$ with intensity function $Z_{\xi}$ and $X^{\xi}$ is a GSNCP driven by

$$
Z^{\xi}(\eta)=\sum_{(c, b, \gamma) \in \Phi^{\left(c_{\xi}, b_{\xi}, \gamma_{\xi}\right)}} \gamma k_{b}(c, \eta), \quad \eta \in \mathbb{R}^{d}
$$

where $\Phi^{(c, b, \gamma)}$ denotes a point process with law $P_{(c, b, \gamma)}^{!}$.

Proposition 3. For Lebesgue almost all $\xi \in \mathbb{R}^{d}$ with $\rho(\xi)>0$,

$$
P_{\xi}^{!}(F)=P\left(X^{\xi} \cup X_{\xi} \in F\right), \quad F \in \mathcal{N}_{\mathrm{lf}} .
$$

Proof. By (23) and arguing as in the proof of Proposition 2 in [29], it suffices to show that

$$
\mathrm{E} \sum_{\xi \in X} \mathbf{1}[\xi \in A,(X \backslash\{\xi\}) \cap B=\emptyset]=\int_{A} P\left(\left(X^{\xi} \cup X_{\xi}\right) \cap B=\emptyset\right) \rho(\xi) \mathrm{d} \xi
$$


for bounded $A, B \in \mathcal{B}^{d}$. It follows also from Proposition 2 in [29] that the left hand side in (26) is given by

$$
\int_{A} \mathrm{E} \sum_{(c, b, \gamma) \in \Phi} \gamma k_{b}(c, \xi) \exp \left(-\int_{B} \sum_{\left(c^{\prime}, b^{\prime}, \gamma^{\prime}\right) \in \Phi} \gamma^{\prime} k_{b^{\prime}}\left(c^{\prime}, \eta\right) \mathrm{d} \eta\right) \mathrm{d} \xi .
$$

By (24),

$$
\begin{aligned}
& \mathrm{E} \sum_{(c, b, \gamma) \in \Phi} \gamma k_{b}(c, \xi) \exp \left(-\int_{B} \sum_{\left(c^{\prime}, b^{\prime}, \gamma^{\prime}\right) \in \Phi} \gamma^{\prime} k_{b^{\prime}}\left(c^{\prime}, \eta\right) \mathrm{d} \eta\right) \\
= & \iint \gamma k_{b}(c, \xi) \exp \left(-\int_{B} \sum_{\left(c^{\prime}, b^{\prime}, \gamma^{\prime}\right) \in \phi \cup\{(c, b, \gamma)\}} \gamma^{\prime} k_{b^{\prime}}\left(c^{\prime}, \eta\right) \mathrm{d} \eta\right) \mathrm{d} P_{(c, b, \gamma)}^{!}(\phi) \mathrm{d} \zeta(c, b, \gamma) \\
= & \int \gamma k_{b}(c, \xi) \exp \left(-\int_{B} \gamma k_{b}(c, \eta) \mathrm{d} \eta\right) \mathrm{E} \exp \left(-\int_{B} \sum_{\left(c^{\prime}, b^{\prime}, \gamma^{\prime}\right) \in \Phi(c, b, \gamma)} \gamma^{\prime} k_{b^{\prime}}\left(c^{\prime}, \eta\right) \mathrm{d} \eta\right) \\
= & \mathrm{d} \zeta(c, b, \gamma) \\
& (\xi) P\left(\left(X^{\xi} \cup X_{\xi}\right) \cap B=\emptyset\right)
\end{aligned}
$$

where we have used the conditional independence of $X^{(\xi)}$ and $X_{\xi}$ given $\left(c_{\xi}, b_{\xi}, \gamma_{\xi}\right)$ to obtain the last equality. Thereby (26) is obtained.

Remark 1. The complication in using Proposition 3 lies in the need of determining $P_{(c, b, \gamma)}^{!}$. This reduced Palm distribution is particular simple for SNCPs, see Proposition 2 in [29]. Suppose that $\Phi$ is a Cox process such that $\Phi \mid \theta$ is a Poisson process with intensity function $\lambda_{\theta}$, where $\theta$ is a random variable. Then

$$
\int h(\phi) \mathrm{d} P_{(c, b, \gamma)}^{!}(\phi)=\mathrm{E}\left[h(\Phi) \lambda_{\theta}(c, b, \gamma)\right] / \lambda(c, b, \gamma)
$$

for $h \geq 0$ measurable, provided $\lambda(c, b, \gamma) \equiv \mathrm{E} \lambda_{\theta}(c, b, \gamma) \in(0, \infty)$. This follows from first conditioning on $\theta$ in the left hand side of (24) and using the Slivnyak-Mecke theorem, and next by taking expectation and then conditioning on $\Phi$. Suppose instead that $\Phi_{\text {cent }}$ is a stationary Markov point process (see Example 4) with intensity $\rho_{\text {cent }}>0$. If we assume for simplicity that the $\left(b_{j}, \gamma_{j}\right)=(b, \gamma)$ are all equal and fixed, then $P_{c}^{!}=P_{(c, b, \gamma)}^{!}$ is concentrated on $N_{\text {lf }}$ and satisfies

$$
\int h(\phi) \mathrm{d} P_{c}^{!}(\phi)=\mathrm{E}\left[h\left(\Phi_{\text {cent }}\right) \lambda^{*}\left(\Phi_{\text {cent }}, c\right)\right] / \rho_{\text {cent }} .
$$


This follows from equation (28) in [29] (which, incidentally, should be corrected by replacing $\tilde{\lambda}(x, \xi)$ with $\tilde{\lambda}(x, \xi) / \tilde{\rho}(\xi))$.

We now consider the $J$-function for a stationary GSNCP with intensity $\rho \in(0, \infty)$, assuming that $k_{1}(c, \xi)=k_{1}(\xi-c)$ and that (7) holds.

Corollary 1. Under the assumptions above, for all $r \geq 0$,

$$
J(r)=\frac{1}{\rho I_{1}(r)} \iint I_{2}(c, b, \gamma, r) I_{3}(c, b, \gamma, r) \mathrm{d} c \mathrm{~d} \chi(b, \gamma)
$$

where

$$
\begin{gathered}
I_{1}(r)=\mathrm{E} \exp \left(-\sum_{j} \int_{b\left(c_{j}, r\right)} \gamma_{j} k_{b_{j}}(\xi) \mathrm{d} \xi\right), \\
I_{2}(c, b, \gamma, r)=\gamma k_{b}(-c) \exp \left(-\int_{b(c, r)} \gamma k_{b}(\eta) \mathrm{d} \eta\right),
\end{gathered}
$$

and

$$
I_{3}(c, b, \gamma, r)=\operatorname{Eexp}\left(-\sum_{\left(c^{\prime}, b^{\prime}, \gamma^{\prime}\right) \in \Phi^{(c, b, \gamma)}} \int_{b\left(c^{\prime}, r\right)} \gamma^{\prime} k_{b^{\prime}}(\xi) \mathrm{d} \xi\right) .
$$

Proof. By definition,

$$
J(r)=P_{0}^{!}\left(\left\{x \in N_{\mathrm{lf}}: x \cap b(0, r)=\emptyset\right\}\right) / P(X \cap b(0, r)=\emptyset) .
$$

Hence by Proposition 3,

$$
\begin{aligned}
& J(r)=P\left(\left(X^{0} \cup X_{0}\right) \cap b(0, r)=\emptyset\right) / P(X \cap b(0, r)=\emptyset) \\
= & \frac{1}{\rho P(X \cap b(0, r)=\emptyset)} \iint P\left(X^{(0)} \cap b(0, r)=\emptyset \mid\left(c_{0}, b_{0}, \gamma_{0}\right)=(c, b, \gamma)\right) \\
& \times P\left(X_{0} \cap b(0, r)=\emptyset \mid\left(c_{0}, b_{0}, \gamma_{0}\right)=(c, b, \gamma)\right) \gamma k_{b}(-c) \mathrm{d} c \mathrm{~d} \chi(b, \gamma) .
\end{aligned}
$$

Since the latter three probabilities are equal to $I_{1}(r), I_{2}(c, b, \gamma, r)$, and $I_{3}(c, b, \gamma, r)$, respectively, we obtain (29).

Example 7. It is well-known that for stationary Poisson cluster processes and SNCPs, $J(r) \leq 1$ and $J$ is non-increasing [21, 29]. Below we show that these properties hold for certain GSNCPs. We let the situation be as in Corollary 1 , and recall that $\mathrm{d} \zeta(c, b, \gamma)=$ $\mathrm{d} c \mathrm{~d} \chi(b, \gamma)$. Notice that if for $\zeta$ almost all $(c, b, \gamma)$ and $r>0$,

$$
I_{1}(r) \geq I_{3}(c, b, \gamma, r)
$$


then (20) and (29) imply that $J(r)<1$ for $r>0$.

If $\Phi$ is a Poisson process so that $\Phi_{\text {cent }}$ is stationary, then $I_{3}(c, b, \gamma, r)=I_{1}(r)$, and so by $(29)$,

$$
J(r)=\frac{1}{\rho} \iint \gamma k_{b}(-c) \exp \left(-\int_{b(c, r)} \gamma k_{b}(\eta) \mathrm{d} \eta\right) \mathrm{d} c \mathrm{~d} \chi(b, \gamma) .
$$

This is slightly extending Corollary 1 in [29] (where, incidentally, there is a minor typos: $k(c)$ should read $k(-c)$ ). It follows that $J$ is non-increasing and $J(r)<1$ for $r>0$.

Consider instead a generalised Neyman-Scott process: Suppose that $\Phi_{\text {cent }}$ is a mixed Poisson process driven by a positive random variable $\theta$ (i.e. $\Phi_{\text {cent }} \mid \theta$ is a stationary Poisson process with intensity $\theta)$, where $\left(\theta, \Phi_{\text {cent }}\right)$ is independent of the $\left(b_{j}, \gamma_{j}\right)$, which are i.i.d. with distribution $Q$ (case (iii) in Example 2). In other words, $\Phi$ is a Cox process driven by a random measure $\Lambda$ given by $\mathrm{d} \Lambda(c, b, \gamma)=\theta \mathrm{d} c \mathrm{~d} Q(b, \gamma)$. Let $\pi$ denote the distribution of $\theta$ and define another positive random variable $\theta^{\prime}$ with distribution

$$
\pi^{\prime}(A)=\frac{1}{\mathrm{E} \theta} \int_{A} \theta \mathrm{d} \pi(\theta)
$$

for Borel sets $A \subseteq(0, \infty)$. Suppose that

$$
\pi \leq_{\text {st }} \pi^{\prime}
$$

(usual stochastic order), i.e. $\pi((t, \infty)) \leq \pi^{\prime}((t, \infty))$ for all $t>0$. For instance, (31) is satisfied if $\pi$ is a gamma distribution. We claim that (31) implies (30): Since $\Phi$ is a Cox process, a slight modification of (27) implies that

$$
\begin{aligned}
I_{3}(c, b, \gamma, r) & =\mathrm{E}\left[\exp \left(-\sum_{j} \int_{b\left(c_{j}, r\right)} \gamma_{j} k_{b_{j}}(\xi) \mathrm{d} \xi\right) \theta\right] / \mathrm{E} \theta \\
& =\operatorname{E} \exp \left(-\sum_{j} \int_{b\left(c_{j}^{\prime}, r\right)} \gamma_{j} k_{b_{j}}(\xi) \mathrm{d} \xi\right)
\end{aligned}
$$

where $\Phi^{\prime}=\left\{\left(c_{j}^{\prime}, b_{j}, \gamma_{j}\right)\right\}$ is a Cox process driven by $\Lambda^{\prime}$ given by $\mathrm{d} \Lambda^{\prime}(c, b, \gamma)=\theta^{\prime} \mathrm{d} c \mathrm{~d} Q(b, \gamma)$. For locally finite measures $\mu_{1}$ and $\mu_{2}$ on $\Omega$, define a partial order $\preceq$ by

$$
\mu_{1} \preceq \mu_{2} \quad \text { whenever } \mu_{1}(B) \leq \mu_{2}(B) \text { for all bounded Borel sets } B \subset \Omega \text {. }
$$


Further, let $\subseteq_{\text {st }}$ denote the usual stochastic order for locally finite random measures $\Gamma_{1}$ and $\Gamma_{2}$ on $\Omega$, i.e.

$\Gamma_{1} \subseteq_{\text {st }} \Gamma_{2} \Leftrightarrow \operatorname{E} f\left(\Gamma_{1}\right) \leq \operatorname{E} f\left(\Gamma_{2}\right) \quad$ whenever $f$ is increasing with respect to $\preceq$.

By (31), $\Lambda \subseteq_{\text {st }} \Lambda^{\prime}$, and so by Theorem 7.4.7 in [34], $\Phi \subseteq_{\text {st }} \Phi^{\prime}$. Now, $I_{3}(c, b, \gamma, r)=I_{3}(r)$ depends only on $r$ and it is of the same form as $I_{1}(r)$ except that $\Phi_{\text {cent }}$ is replaced by $\Phi_{\text {cent }}^{\prime}$. Consequently (30) holds, since the function

$$
f(\mu)=\exp \left(-\sum_{(c, b, \gamma) \in \mu} \int_{b(c, r)} \gamma k_{b}(\xi) \mathrm{d} \xi\right),
$$

where $\mu$ is a locally finite measure on $\Omega$, is non-increasing with respect to $\preceq$, and $I_{1}(r)=\mathrm{E} f(\Phi)$ and $I_{3}(r)=\mathrm{E} f\left(\Phi^{\prime}\right)$.

Consider next a generalised shot noise G Cox process as in Example 3 where we assume that $\tau$ is a random variable with distribution $\pi,(\alpha, \kappa)$ is fixed, the $b_{j}$ are i.i.d. with distribution $\nu$, and they are independent of $\left(\tau,\left\{\left(c_{j}, \gamma_{j}\right)\right\}\right)$. Then the Cox process $\Phi$ is driven by $\Lambda$ given by $\mathrm{d} \Lambda(c, b, \gamma)=\lambda_{\tau}(\gamma) \mathrm{d} c \mathrm{~d} \nu(b) \mathrm{d} \gamma$, where $\lambda_{\tau}(\gamma)=\lambda_{\theta}(\gamma)$ is given by (10). We have that

$$
I_{3}(c, b, \gamma, r)=\mathrm{E}\left[\exp \left(-\sum_{j} \int_{b\left(c_{j}, r\right)} \gamma_{j} k_{b_{j}}(\xi) \mathrm{d} \xi\right) \lambda_{\tau}(\gamma)\right] / \mathrm{E} \lambda_{\tau}(\gamma) .
$$

So arguing as above for a generalised Neyman-Scott process, (30) holds if $\pi_{\gamma} \leq_{\mathrm{st}} \pi$ for any $\gamma>0$, where

$$
\pi_{\gamma}(A)=\int_{A} \lambda_{\tau}(\gamma) \mathrm{d} \pi(\tau) / \int \lambda_{\tau}(\gamma) \mathrm{d} \pi(\tau)
$$

for Borel sets $A \subseteq(0, \infty)$. For instance, if $\tau$ is gamma distributed, then $\pi_{\gamma} \leq_{\text {st }} \pi$ for any $\gamma>0$.

Finally, consider a GSNCP where $\Phi_{\text {cent }}$ is a stationary Markov point process on $\mathbb{R}^{d}$ with intensity $\rho_{\text {cent }} \in(0, \infty)$, and the $\left(b_{j}, \gamma_{j}\right)=(b, \gamma)$ are all equal and fixed. Then (29) becomes

$$
J(r)=\frac{1}{I_{1}(r)} \int I_{2}(c, b, \gamma, r) I_{3}(c, b, \gamma, r) \mathrm{d} c
$$

where

$$
I_{3}(c, b, \gamma, r)=\mathrm{E}\left[\exp \left(-\sum_{j} \int_{b\left(c_{j}, r\right)} \gamma_{j} k_{b}(\xi) \mathrm{d} \xi\right) \frac{\lambda^{*}\left(\Phi_{\text {cent }}, c\right)}{\rho_{\text {cent }}}\right],
$$

cf. (28). However, we do not know how to get any further with this expression. 


\section{Simulation of generalised shot noise Cox processes}

This section considers various simulation algorithms for the restriction $X_{W}=X \cap W$ of $X$ to a Borel set $W \subset \mathbb{R}^{d}$ with volume $|W| \in(0, \infty)$, or for conditional simulation of $\Phi$ given $X_{W}$.

\subsection{Simulation with edge effects and truncation}

Clearly, $X_{W}$ is a Cox process driven by the random field $Z_{W}(\xi)=\mathbf{1}[\xi \in W] Z(\xi)$. For the simulation of $X_{W}$ a truncation may be needed to deal with the possibly infinite point process $\Phi$ entering $Z_{W}$. Assume that $W_{\text {ext }} \subseteq \mathbb{R}^{d}, B \leq \infty$, and $\varepsilon \geq 0$ so that $\zeta(D)$ is finite, where $D=W_{\text {ext }} \times(0, B) \times(\varepsilon, \infty)$. For instance, for the specific example of a generalised shot noise G process in Example 3, the condition that $\varepsilon>0$ is needed if $\mathrm{P}(\alpha \geq 0)>0$.

An approximate simulation of $X_{W}$ is obtained by simulating first the point process $\Phi \cap D$ and next the corresponding independent Poisson processes $X_{j} \cap W$ with intensity functions $\xi \mapsto \gamma_{j} k_{b_{j}}\left(c_{j}, \xi\right) \mathbf{1}[\xi \in W],\left(c_{j}, b_{j}, \gamma_{j}\right) \in \Phi \cap D$. As noticed in [29], in applications edge effects may enter, since $W_{\text {ext }}$ is typically a bounded window such that $W \subset W_{\text {ext }}$. Below we quantify the error of such approximate simulations by extending Proposition 3 in [29].

Let

$$
M_{W}=\sum_{j} \mathbf{1}\left[\left\{c_{j} \notin W_{\text {ext }}\right\} \cup\left\{b_{j} \geq B\right\} \cup\left\{\gamma_{j} \leq \varepsilon\right\}\right] \operatorname{card}\left(X_{j} \cap W\right)
$$

denote the number of missing points when we make an approximate simulation of $X_{W}$ by ignoring clusters $X_{j}$ with $c_{j} \notin W_{\text {ext }}$ or $b_{j} \geq B$ or $\gamma_{j} \leq \varepsilon$. Further, let $q_{W}$ be the probability that some cluster $X_{j}$ with centre $c_{j} \notin W_{\text {ext }}$ or $b_{j} \geq B$ or $\gamma_{j} \leq \epsilon$ has a point in $W$. Finally, assume there exists a function $k_{W}^{\text {dom }}:(0, \infty) \times \mathbb{R}^{d} \times \mathbb{R}^{d} \mapsto[0, \infty)$ satisfying the following conditions.

Condition 1. If $\xi \in W$ then $k_{W}^{\mathrm{dom}}(b, c, \xi) \geq k_{b}(c, \xi)$, and $k_{W}^{\mathrm{dom}}(b, c, \xi)=0$ if $\xi \notin W$.

Condition 2. The integral

$$
a_{W}^{\mathrm{dom}}(c, b)=\int_{W} k_{W}^{\mathrm{dom}}(b, c, \xi) \mathrm{d} \xi, \quad(c, b) \in \mathbb{R}^{d} \times(0, \infty),
$$

can be easily computed. 
Proposition 4. We have that

$$
\mathrm{E} M_{W} \leq \int \mathbf{1}\left[\left\{c \notin W_{\text {ext }}\right\} \cup\{b \geq B\} \cup\{\gamma \leq \varepsilon\}\right] \gamma a_{W}^{\text {dom }}(c, b) \mathrm{d} \zeta(c, b, \gamma)
$$

and

$$
q_{W} \leq 1-\exp \left(-\int \mathbf{1}\left[\left\{c \notin W_{\text {ext }}\right\} \cup\{b \geq B\} \cup\{\gamma \leq \varepsilon\}\right] \gamma a_{W}^{\text {dom }}(c, b) \mathrm{d} \zeta(c, b, \gamma)\right) .
$$

Proof. The proof of (32) is similar to that of Proposition 3 in [29]. Conditional on $\Phi$, the clusters $X_{j}$ are independent Poisson processes with intensity functions $\gamma_{j} k_{b_{j}}\left(c_{j}, \cdot\right)$, so

$$
q_{W}=1-\mathrm{E} \prod_{j}\left[\exp \left(-\gamma_{j} \int_{W} k_{b_{j}}\left(c_{j}, \xi\right) \mathrm{d} \xi\right)\right]^{\mathbf{1}\left[\left\{c_{j} \notin W_{\mathrm{ext}}\right\} \cup\left\{b_{j} \geq B\right\} \cup\left\{\gamma_{j} \leq \epsilon\right\}\right]} .
$$

Hence, by Jensen's inequality and (5),

$$
\begin{aligned}
q_{W} & \leq 1-\exp \left(-\mathrm{E} \sum_{j} \mathbf{1}\left[\left\{c_{j} \notin W_{\text {ext }}\right\} \cup\left\{b_{j} \geq B\right\} \cup\left\{\gamma_{j} \leq \epsilon\right\}\right] \gamma_{j} \int_{W} k_{b_{j}}\left(c_{j}, \xi\right) \mathrm{d} \xi\right) \\
& =1-\exp \left(-\int \mathbf{1}\left[\left\{c \notin W_{\text {ext }}\right\} \cup\{b \geq B\} \cup\{\gamma \leq \epsilon\}\right] \gamma \int_{W} k_{b}(c, \xi) \mathrm{d} \xi \mathrm{d} \zeta(c, b, \gamma)\right) .
\end{aligned}
$$

Thereby, using Conditions 1 and 2, (33) is obtained.

Remark 2. When $\Phi$ is a Poisson process, (33) can be improved, cf. [33]. Indeed, using (34), Conditions 1 and 2, and arguments similar to the derivation of the generating functional of Poisson processes (see [33]), we obtain that

$$
\begin{gathered}
1-q_{W}=\exp \left[-\int \mathbf{1}\left[\left\{c \notin W_{\text {ext }}\right\} \cup\{b \geq B\} \cup\{\gamma \leq \epsilon\}\right]\right. \\
\left.\times\left(1-\exp \left(-\gamma \int k_{b}(c, \xi) \mathrm{d} \xi\right)\right) \mathrm{d} \zeta(c, b, \gamma)\right] \\
\geq \exp \left[-\int \mathbf{1}\left[\left\{c \notin W_{\text {ext }}\right\} \cup\{b \geq B\} \cup\{\gamma \leq \epsilon\}\right]\left(1-\exp \left(-\gamma a_{W}^{\text {dom }}(b, c)\right) \mathrm{d} \zeta(c, b, \gamma)\right)\right] .
\end{gathered}
$$

Note also that by the coupling inequality (see [23]), the upper bound on $q_{W}$ in (33) is also an upper bound on the total variation distance between the law of $X_{W}$ and the law of the truncated process.

Example 8. Suppose that (7) holds. For specific models of $\chi$ and $k_{1}$, the upper bounds in Proposition 4 can be calculated along similar lines as in [6, 29]. For example, let 
$W=b(0, R)$ and $W_{\text {ext }}=b(0, R+r)$, where $R$ and $r$ are two positive constants, and let $k_{1}$ be the Gaussian kernel (3). Define $k_{W}^{\operatorname{dom}}(b, \xi-c)=\sup _{\eta \in W} k_{b}(\eta-c)$ for $\xi \in W$, and set $\sigma_{d}=2 \pi^{d / 2} / \Gamma(d / 2)$. Proceeding as in Example 5 in [29],

$$
a(\|c\|, b) \equiv a_{W}^{\operatorname{dom}}(c, b)=\left[\frac{\omega_{d} R^{d}}{\left(2 \pi b^{2}\right)^{d / 2}}\right] \exp \left[-\mathbf{1}[\|c\|>R] \frac{(\|c\|-R)^{2}}{2 b^{2}}\right]
$$

and the integral in (32) and (33) is given by

$$
\sigma_{d} \int_{0}^{\infty} \int_{0}^{\infty} \gamma \mathrm{d} \chi(b, \gamma) \int_{0}^{\infty} s^{d-1} a(s, b) \mathrm{d} s-\sigma_{d} \int_{0}^{B} \int_{\varepsilon}^{\infty} \gamma \mathrm{d} \chi(b, \gamma) \int_{0}^{R+r} s^{d-1} a(s, b) \mathrm{d} s .
$$

This may be determined by numerical methods for specific models of generalised Neyman-Scott and generalised shot noise G Cox processes, cf. [6, 29].

\subsection{Simulation without edge effects and truncation}

Perfect simulation of $X_{W}$ can be obtained by independent thinning of the nonempty clusters in a Cox process $X_{W}^{\text {dom }}$ driven by

$$
Z_{W}^{\mathrm{dom}}(\xi)=\sum_{j} \gamma_{j} k_{W}^{\mathrm{dom}}\left(b_{j}, c_{j}, \xi\right)
$$

where $k_{W}^{\text {dom }}$ satisfies Conditions 1 and 2. The details for SNCPs are carefully discussed in $[6,29,33]$, so in this section we give only a description of the algorithm and discuss some applications for GSNCPs.

Let $X_{j}^{\text {dom }},\left(c_{j}, b_{j}, \gamma_{j}\right) \in \Phi$, denote the clusters of $X_{W}^{\text {dom }}$, and let $\Phi_{W}^{\text {dom }}=\left\{\left(c_{j}, b_{j}, \gamma_{j}\right) \in\right.$ $\left.\Phi: X_{j}^{\text {dom }} \neq \emptyset\right\}$. In addition to Conditions 1 and 2 , assume that the following condition holds.

Condition 3. It is feasible to simulate $\Phi_{W}^{\mathrm{dom}}$ (hence $\Phi_{W}^{\mathrm{dom}}$ is almost surely finite).

Example 9 below demonstrates that to check Condition 3 will depend very much on the choice of model for $\Phi$, the kernel $k_{1}$, etc.

\section{Perfect simulation algorithm for GSNCPs.}

(a) Generate the point process $\Phi_{W}^{\mathrm{dom}}=\left\{\left(c_{1}, b_{1}, \gamma_{1}\right), \ldots,\left(c_{N}, b_{N}, \gamma_{N}\right)\right\}$.

(b) For each $j=1, \ldots, N$, generate

(i) $X_{j}^{\text {dom }}$, which is distributed as a conditional Poisson process with intensity function $\gamma_{j} k_{W}^{\mathrm{dom}}\left(b_{j}, c_{j}, \cdot\right)$ given that it is nonempty; 
(ii) $X_{j}^{\prime}$, which is an independent thinning of $X_{j}^{\text {dom }}$ with retention probabilities $k_{b_{j}}\left(c_{j}, \xi\right) / k_{W}^{\mathrm{dom}}\left(b_{j}, c_{j}, \xi\right)$ for $\xi \in X_{j}^{\text {dom }}$.

(c) Return $\bigcup_{j} X_{j}^{\prime}$.

For the loop in (b), it is implicit that the generation of processes in (i) and (ii) is independent of previous generations. The output in (c) follows the same distribution as $X_{W}$, see e.g. Proposition 4 in [29].

Example 9. For the arguments below, it is useful to notice that if $\Phi$ is a Cox process, we can obtain $\Phi_{W}^{\mathrm{dom}}$ by independent thinning of $\Phi$ with retention probabilities

$$
p_{W}^{\mathrm{dom}}(c, b, \gamma)=1-\exp \left(-\gamma a_{W}^{\mathrm{dom}}(c, b)\right), \quad(c, b, \gamma) \in \Omega .
$$

Suppose that $\Phi$ is a Poisson process. Then clearly $\Phi_{W}^{\mathrm{dom}}$ is a Poisson process on $\Omega$, with intensity measure

$$
\zeta_{W}^{\mathrm{dom}}(D)=\int_{D} p_{W}^{\mathrm{dom}}(c, b, \gamma) \zeta(c, b, \gamma)
$$

Condition 3 means that $\zeta_{W}^{\text {dom }}(\Omega)<\infty$ and we are able to generate $\Phi_{W}^{\text {dom }}$. Example 6 in [29] gives a detailed discussion of this condition for SNCP, and this discussion easily extends to the present case.

Suppose instead that $\Phi$ is a generalised Neyman-Scott process, where the $\left(b_{j}, \gamma_{j}\right)$ are i.i.d. with distribution $Q$, and $\Phi_{\text {cent }}$ is a Cox process driven by a random field $Y(\cdot)$, which is bounded by a constant $M$ and independent of the $\left(b_{j}, \gamma_{j}\right)$. Further, let $k_{1}, W$, $W_{\text {ext }}, k_{W}^{\mathrm{dom}}$, and $a(\|c\|, b)=a_{W}^{\mathrm{dom}}(c, b)$ be defined as in Example 8. Then $\Phi_{W}^{\mathrm{dom}}$ is a Cox process on $\Omega$ driven by the random measure $\mathrm{d} m(c, b, \gamma)=p_{W}^{\mathrm{dom}}(c, b, \gamma) Y(c) \mathrm{d} c \mathrm{~d} Q(b, \gamma)$. So

$$
\mathrm{E} \Phi_{W}^{\mathrm{dom}}=\mathrm{E} m(\Omega) \leq M \sigma_{d} \int_{0}^{\infty} \int_{(0, \infty)^{2}} s^{d-1}(1-\exp (-\gamma a(s, b))) \mathrm{d} s \mathrm{~d} Q(b, \gamma) \equiv \beta_{W}^{\mathrm{dom}}
$$

where we assume that $\beta_{W}^{\text {dom }}$ is finite and can be determined by numerical integration (see e.g. page 628 in [29]). At least in principle simulation of $\Phi_{W}^{\text {dom }}$ is straightforward by thinning: First, simulate a Poisson variate $N$ with mean $\beta_{W}^{\text {dom }}$. Second, generate independent points $\left(s_{j} u_{j}, b_{j}, \gamma_{j}\right)$ for $j=1, \ldots, N$, where $u_{j}$ is a uniformly distributed unit vector in $\mathbb{R}^{d},\left(s_{j}, b_{j}, \gamma_{j}\right)$ has distribution

$$
\mathrm{P}(A) \propto \iint \mathbf{1}[(s, b, \gamma) \in A] s^{d-1}[1-\exp (-\gamma a(s, b))] \mathrm{d} s \mathrm{~d} Q(b, \gamma), \quad A \subseteq(0, \infty)^{3},
$$


and $u_{j}$ is independent of $\left(s_{j}, b_{j}, \gamma_{j}\right)$ (here rejection sampling may be useful). Third, simulate $Y\left(s_{j} u_{j}\right)$ for $j=1, \ldots, N$, and assign each $\left(s_{j} u_{j}, b_{j}, \gamma_{j}\right)$ to $\Phi_{W}^{\text {dom }}$ with probability $Y\left(s_{j} u_{j}\right) / M$ (where these assignments are independent).

Similar ideas apply for a generalised shot noise G Cox process: Consider the case in Example 3 where the $b_{j}$ are i.i.d. with distribution $\nu$, and let again $k_{1}$, $W$, etc. be as in Example 8. Then $\Phi_{W}^{\text {dom }}$ is a Cox process driven by the random measure $\mathrm{d} m_{\theta}(c, b, \gamma)=p_{W}^{\mathrm{dom}}(c, b, \gamma) \lambda_{\theta}(\gamma) \mathrm{d} c \mathrm{~d} \nu(b) \mathrm{d} \gamma$, where $\lambda_{\theta}$ is given by (10). So $\Phi_{W}^{\mathrm{dom}}$ is almost surely finite if

$$
\mathrm{E} \Phi_{W}^{\mathrm{dom}}=\sigma_{d} \int_{0}^{\infty} \int_{0}^{\infty} \int_{0}^{\infty} s^{d-1}(1-\exp (-\gamma a(s, b))) \mathrm{E} \lambda_{\theta}(\gamma) \mathrm{d} s \mathrm{~d} \nu(b) \mathrm{d} \gamma
$$

is finite, and we can then at least in principle simulate $\Phi_{W}^{\mathrm{dom}}$ as follows. Generate first $\theta$ and next $\Phi_{W}^{\text {dom }}$ as a Poisson process with intensity measure $m_{\theta}$.

Finally, consider the Markov point process setting in Example 4, where for simplicity we assume that $\Phi_{\text {cent }}$ is a finite Markov point process contained in $W_{\text {ext }}$ which is bounded. We cannot exploit the same ideas as above, since $\Phi_{\text {cent }}$ is not easily viewed as a Cox process. However, dominated coupling from the past [18] may be used for making a perfect simulation of $\Phi_{\text {cent }}$. Then, since $\Phi_{\text {cent }}$ is independent of the $\left(b_{j}, \gamma_{j}\right)$, which are either i.i.d. or identical (cf. Example 4), it may be straightforward to simulate $\Phi_{W}^{\mathrm{dom}}$.

\subsection{Conditional simulation}

Assume that $X_{W}=x=\left\{x_{1}, \ldots, x_{m}\right\} \neq \emptyset$ is observed. Simulation from the conditional distribution of $\Phi$ given $X_{W}=x$ is needed for predicting $\Phi$ and also for performing likelihood and Bayesian inference based on MCMC methods, cf. Section 4.3 in [29] and the references therein. In this section firstly, following [14] (see also [27, 33]), we describe the birth-death-move Metropolis-Hastings algorithm for conditional simulation of the process $\Phi \mid X_{W}=x$. Secondly, we give sufficient conditions which guarantee geometric ergodicity of the algorithm (or in fact $V$-uniform; the reader is referred to [26] or Section 7.2 in [33] for background material on Markov chains).

Throughout this section we assume the following. The process $\Phi$ is almost surely finite and contained in $D=W_{\text {ext }} \times B \times(\varepsilon, \infty)$, with $W \subseteq W_{\text {ext }} \subseteq \mathbb{R}^{d}, B \subseteq(0, \infty)$, and $\varepsilon \geq 0$. We will usually have that $W_{\text {ext }}$ is bounded, while depending on the context 
it may be natural to consider cases where $B$ is a bounded or unbounded interval. The case where $\varepsilon$ is positive will first be needed for technical reasons when establishing geometric ergodicity. Further, $\nu$ denotes a Poisson process on $D$ with a diffuse intensity measure $\mu$ such that $0<\mu(D)<\infty$. Finally, $\Phi$ has a density $p$ with respect to $\nu$.

Then $X_{W} \mid \Phi$ is a Poisson process and it has a density with respect to a unit rate Poisson process on $W$,

$$
f(x \mid \Phi)=\exp \left(|W|-\int_{W} Z(\xi) \mathrm{d} \xi\right) \prod_{\xi \in x} Z(\xi) .
$$

Hence, an unnormalised density for $\Phi \mid X_{W}=x$ with respect to $\nu$ is given by

$$
\pi(\phi \mid x)=f(x \mid \phi) p(\phi)
$$

where the normalising constant is unknown but depends only on the data $x$.

We turn now to the description of the birth-death-move Metropolis-Hastings algorithm. It generates a Markov chain defined on

$$
\Omega_{x}=\{\phi \subset D: \operatorname{card}(\phi)<\infty, \pi(\phi \mid x)>0\} .
$$

Let $0<q_{1}<1$ and $0<q_{2} \leq 1$ be given numbers, and for $\phi \in \Omega_{x}$ and $(c, b, \gamma) \in D$, define

$$
r[\phi,(c, b, \gamma)]=\frac{\left(1-q_{1}\right) \pi(\phi \cup\{(c, b, \gamma)\} \mid x) \mu(D)}{q_{1} \pi(\phi \mid x)(\operatorname{card}(\phi)+1)} .
$$

If $\phi=\left\{\left(c_{1}, b_{1}, \gamma_{1}\right), \ldots,\left(c_{n}, b_{n}, \gamma_{n}\right)\right\} \in \Omega_{x}$ is the current state of the chain, the next state is generated as follows:

- with probability $q_{1} q_{2}$ make a birth step:

- generate $(c, b, \gamma)$ with law $\mu / \mu(D)$

- with probability $\min \{1, r[\phi,(c, b, \gamma)]\}$ return $\phi \cup\{(c, b, \gamma)\}$ as the next state

- if $n>0$, with probability $\left(1-q_{1}\right) q_{2}$ make a death step:

- generate $i$ according to the uniform law on $\{1, \ldots, n\}$

- with probability $\min \left\{1, r\left[\phi \backslash\left\{\left(c_{i}, b_{i}, \gamma_{i}\right)\right\},\left(c_{i}, b_{i}, \gamma_{i}\right)\right]^{-1}\right\}$ return $\phi \backslash\left\{\left(c_{i}, b_{i}, \gamma_{i}\right)\right\}$ as the next state

- if $n>0$, with probability $1-q_{2}$ make a move step: 
- generate $i$ according to the uniform law on $\{1, \ldots, n\}$

- generate $(c, b, \gamma)$ with law $\mu / \mu(D)$

- with probability $\min \left\{1, \pi\left(\left(\phi \backslash\left\{\left(c_{i}, b_{i}, \gamma_{i}\right)\right\}\right) \cup\{(c, b, \gamma)\} \mid x\right) / \pi(\phi \mid x)\right\}$ return $\left(\phi \backslash\left\{\left(c_{i}, b_{i}, \gamma_{i}\right)\right\}\right) \cup\{(c, b, \gamma)\}$ as the next state

- else return $\phi$ as the next state.

Assuming that the initial state is in $\Omega_{x}$, the chain stays in $\Omega_{x}$. Note that the empty point configuration is not contained in $\Omega_{x}$, since $x \neq \emptyset$. Further, the chain is reversible with invariant (unnormalised) density $\pi(\cdot \mid x)$. This follows along similar lines as in the proofs of Propositions 7.11, 7.12, and 7.15 in [33].

We consider two situations where irreducibility of the chain is satisfied. If for any $\phi \in \Omega_{x}, \phi^{\prime} \in \Omega_{x}, \psi \subset \phi$, and $\psi^{\prime} \subset \phi^{\prime}$, we have that $\phi \cup \psi^{\prime} \in \Omega_{x}$ and $(\phi \backslash \psi) \cup \phi^{\prime} \in \Omega_{x}$, then irreducibility holds. Briefly this follows because the chain can then move up from $\phi$ to $\phi \cup \phi^{\prime}$ and then down to $\phi^{\prime}$. Note that $f(x \mid \phi)>0$ if $f\left(x \mid \phi^{\prime}\right)>0$ and $\phi^{\prime} \subset \phi$, and that $\pi(\phi \mid x)>0$ if and only if $f(x \mid \phi)>0$ and $p(\phi)>0$. Thus irreducibility holds if for all $\phi \in \Omega_{x}$ and $(c, b, \gamma) \in D$,

$$
p(\phi)>0 \Rightarrow p(\phi \cup\{(c, b, \gamma)\})>0
$$

This condition is satisfied for SNCPs (since $p=1$ in [29]). However, (36) is not always satisfied for other models of interests. For example, if a hard core condition is imposed so that $p(\phi \cup\{(c, b, \gamma)\})=0$ if a cluster centre from $\phi$ is sufficiently close to $c$, then (36) is violated. In fact, the opposite will often hold: $p$ is said to be hereditary on $\Omega_{x}$ if for all $\phi \in \Omega_{x}$ and $(c, b, \gamma) \in D$,

$$
p(\phi \cup\{(c, b, \gamma)\})>0 \Rightarrow p(\phi)>0
$$

In that case we need to impose further conditions: If $q_{2}<1$ (i.e. moves are possible),

$$
k_{1}\left(c, x_{i}\right)>0 \quad \text { for all } c \in W_{\text {ext }} \text { and } i=1, \ldots, m
$$

(i.e. $f(x \mid \phi)>0$ for all finite non-empty $\phi \subset D$ ), and (37) hold, then we have irreducibility. Briefly, this follows since $\pi(\cdot \mid x)$ is now hereditary on $\Omega_{x}$, so if $\phi \in \Omega_{x}$, $\phi^{\prime} \in \Omega_{x},(c, b, \gamma) \in \phi$, and $\left(c^{\prime}, b^{\prime}, \gamma^{\prime}\right) \in \phi^{\prime}$, the chain can first move down from $\phi$ to $(c, b, \gamma)$, then move to $\left(c^{\prime}, b^{\prime}, \gamma^{\prime}\right)$, and finally move up to $\phi^{\prime}$. Note that $(38)$ is clearly 
satisfied for a positive kernel such as the Gaussian kernel (3), but (38) may easily be violated for the uniform kernel (4).

In the sequel irreducibility is assumed. Note that the chain is clearly aperiodic, since it can be staying in the same state for one or more transitions. Below stronger conditions than those above will be assumed to establish $V$-uniform ergodicity. We consider the cases with and without moves separately (i.e. the two cases $q_{2}<1$ and $\left.q_{2}=1\right)$.

The following Proposition 5 concerns the case $q_{2}=1$, in which case we refer to our Metropolis-Hastings algorithm as the birth-death algorithm. The proposition encompasses Proposition 5 in [29] (where the $b_{j}$ are equal and fixed, $\Phi$ is a Poisson process, and $p=1$ ). The following conditions are assumed to hold.

(a) $p$ is locally stable, i.e. there is a finite constant $\Lambda$ so that

$$
p(\phi \cup\{(c, b, \gamma)\}) \leq \Lambda p(\phi)
$$

for all finite $\phi \subset D$ and $(c, b, \gamma) \in D$.

(b) For any positive integer $K$ there exists a positive function $h_{K}$ such that

$$
h_{K}(c, b, \gamma) p(\phi) \leq p(\phi \cup\{(c, b, \gamma)\})
$$

for all $(c, b, \gamma) \in D$ and $\phi \in \Omega_{x}$ for which $\operatorname{card}(\phi) \leq K$.

(c) There exists a positive constant $\delta>0$ such that

$$
\int_{W} k_{b}(c, \xi) \mathrm{d} \xi \geq \delta
$$

for any $(c, b) \in W_{\text {ext }} \times B$.

(d) For $i=1, \ldots, m$,

$$
\int \mathbf{1}\left[k_{b}\left(c, x_{i}\right)>0\right] \mathrm{d} \mu(c, b, \gamma)>0 .
$$

(e) There exist positive constants $\delta_{1}^{\prime}, \ldots, \delta_{m}^{\prime}, \delta_{1}^{\prime \prime}, \ldots, \delta_{m}^{\prime \prime}$ such that

$$
\delta_{i}^{\prime} \leq k_{b}\left(c, x_{i}\right) \leq \delta_{i}^{\prime \prime} \quad \text { whenever } k_{b}\left(c, x_{i}\right)>0 \text { and }(c, b) \in W_{\text {ext }} \times B .
$$

Conditions (a) and (b) are automatically satisfied for SNCPs (as $p=1$ ). For GSNCPs, condition (b) implies (36), and so irreducibility is ensured. Conditions (a)-(e) are further discussed in Example 10 below. 
Proposition 5. Assume that $q_{2}=1, \varepsilon>0$, and conditions (a)-(e) are satisfied. Then for any function $V(\phi)=\beta^{\operatorname{card}(\phi)}, \phi \in \Omega_{x}$, with $\beta>1$, the birth-death algorithm is $V$-uniformly ergodic.

Proof. We show first that for any positive integer $N$, the set $S_{N}=\left\{\phi \in \Omega_{x}\right.$ : $\operatorname{card}(\phi) \leq N\}$ is a small set. This means that $P^{t}(\phi, F) \geq \varepsilon^{\prime} Q(F)$ for any $\phi \in S_{N}$ and any measurable set $F \subseteq \Omega_{x}$, where $\varepsilon^{\prime}>0$ is some positive constant, $Q$ is some non-zero measure on $\Omega_{x}$, and $P^{t}(\phi, \cdot)$ denotes the $t$-step transition probability of the Metropolis-Hastings chain when it starts in $\phi$.

We shall use the following bounds. Let $\phi \in \Omega_{x}$ and $(c, b, \gamma) \in D$. Arguing as at the beginning of the proof of Proposition 5 in [29], using (a), (c), and (e), it is seen that

$$
\exp \left(-\gamma / b^{d}\right) p(\phi \cup\{(c, b, \gamma)\}) / p(\phi) \leq \pi(\phi \cup\{(c, b, \gamma)\} \mid x) / \pi(\phi \mid x) \leq M
$$

where $M>0$ is a constant. Further, $0<\mu(D)<\infty$ implies that for any positive integer $K$, there exist a constant $L_{K}>\varepsilon$ and a non-void interval $B_{K} \subseteq B$ such that $\rho_{K}=$ $\inf B_{K} \in(0, \infty),\left(\left(1-q_{1}\right) / q_{1}\right) \exp \left(-L_{K} / \rho_{K}^{d}\right) \mu(D) \Lambda \leq 1$ and $\mu\left(W_{\text {ext }} \times B \times\left(\varepsilon, L_{K}\right)\right)>0$.

Furthermore, by (d) and since $h_{K}$ is a positive function,

$$
\int \mathbf{1}\left[k_{b}\left(c, x_{i}\right)>0, b \in B_{K}, \gamma<L_{K}\right] h_{K}(c, b, \gamma) \mathrm{d} \mu(c, b, \gamma)>0, \quad i=1, \ldots, m .
$$

Let $m^{\prime}>\max \left\{m,\left(\left(1-q_{1}\right) / q_{1}\right) M \mu(D)-1\right\}$ be an integer and set $a=\min \left\{q_{1}, 1-q_{1}\right\}$. For any $\phi=\left\{\left(c_{1}, b_{1}, \gamma_{1}\right),\left(c_{2}, b_{2}, \gamma_{2}\right), \ldots\left(c_{k}, b_{k}, \gamma_{k}\right)\right\} \in S_{N}$ and measurable $F \subseteq \Omega_{x}$,

$$
\begin{aligned}
& P^{m^{\prime}+N}(\phi, F) \geq \int_{D} \ldots \int_{D} \int_{D} \ldots \int_{D} \\
& \prod_{l=1}^{m^{\prime}} a \min \left\{1, r\left[\phi \cup\left\{\left(v_{1}, u_{1}, \sigma_{1}\right), \ldots,\left(v_{l-1}, u_{l-1}, \sigma_{l-1}\right)\right\},\left(v_{l}, u_{l}, \sigma_{l}\right)\right]\right\} \\
& \times \prod_{j=1}^{k} \frac{a}{k+m^{\prime}-j+1} \min \left\{1, r\left[\left(\phi \backslash\left\{\left(c_{1}, b_{1}, \gamma_{1}\right), \ldots,\left(c_{j}, b_{j}, \gamma_{j}\right)\right\}\right) \cup\right.\right. \\
& \times \prod_{i=1}^{N-k} a\left(1-\min \left\{1, r\left[\left\{\left(v_{1}, u_{1}, \sigma_{1}\right), \ldots,\left(v_{m^{\prime}}, u_{m^{\prime}}, \sigma_{m^{\prime}}\right)\right\},\left(v_{i}^{\prime}, u_{i}^{\prime}, \sigma_{i}^{\prime}\right)\right]\right\}\right) \\
& \quad \times \mathbf{1}\left[\left\{\left(v_{1}, u_{1}, \sigma_{1}\right), \ldots,\left(v_{m^{\prime}}, u_{m^{\prime}}, \sigma_{m^{\prime}}\right)\right\} \in F\right] \frac{\mathrm{d} \mu\left(v_{1}, u_{1}, \sigma_{1}\right)}{\mu(D)} \cdots \frac{\mathrm{d} \mu\left(v_{m^{\prime}}, u_{m^{\prime}}, \sigma_{m^{\prime}}\right)}{\mu(D)} \\
& \quad \times \frac{\mathrm{d} \mu\left(v_{1}^{\prime}, u_{1}^{\prime}, \sigma_{1}^{\prime}\right)}{\mu(D)} \cdots \frac{\mathrm{d} \mu\left(v_{N-k}^{\prime}, u_{N-k}^{\prime}, \sigma_{N-k}^{\prime}\right)}{\mu(D)}
\end{aligned}
$$


corresponding to first adding $\left(v_{1}, u_{1}, \sigma_{1}\right), \ldots,\left(v_{m^{\prime}}, u_{m^{\prime}}, \sigma_{m^{\prime}}\right)$ to $\phi$, next deleting the points in $\phi$, and finally making no changes when $N-k$ births are proposed. From (35) and the last inequality in (39), we obtain for each of the terms in (42) that $r[\ldots]^{-1} \geq\left(q_{1} /\left(1-q_{1}\right)\right)\left(m^{\prime}+1\right) /(M \mu(D)) \geq 1$, i.e. $\min \left\{1, r[\ldots]^{-1}\right\}=1, j=1, \ldots, k$, and for each of the terms in $(43)$ that $r[\ldots] \leq\left(\left(1-q_{1}\right) / q_{1}\right) M \mu(D) /\left(m^{\prime}+1\right) \leq 1$, i.e. $1-\min \{1, r[\ldots]\}=1-\left(\left(1-q_{1}\right) / q_{1}\right) M \mu(D) /\left(m^{\prime}+1\right), i=1, \ldots, N-k$. Further, combining (35), (b), and the first inequality in (39), we obtain for each of the terms in (41) that

$$
r[\ldots] \geq \frac{\left(1-q_{1}\right) \exp \left(-L_{m^{\prime}+N} / \rho_{m^{\prime}+N}^{d}\right) \mu(D)}{q_{1}(k+l)} h_{m^{\prime}+N}\left(v_{l}, u_{l}, \sigma_{l}\right)
$$

if $\sigma_{l}<L_{m^{\prime}+N}, u_{l} \in B_{m^{\prime}+N}, l=1, \ldots, m^{\prime}$. Therefore,

$$
\begin{aligned}
P^{m^{\prime}+N}(\phi, F) & \geq a^{m^{\prime}+N}\left(\frac{1-q_{1}}{q_{1}}\right)^{m^{\prime}} \frac{\left[\exp \left(-L_{m^{\prime}+N} / \rho_{m^{\prime}+N}^{d}\right) \mu(D)\right]^{m^{\prime}}}{\left[\prod_{l=1}^{m^{\prime}}(k+l)\right]\left[\prod_{j=1}^{k}\left(m^{\prime}+j\right)\right]} \\
& \times\left[1-\frac{\left(1-q_{1}\right) M \mu(D)}{q_{1}\left(m^{\prime}+1\right)}\right]^{N-k} Q(F)
\end{aligned}
$$

where

$$
\begin{aligned}
Q(F)= & \int_{\left(v_{1}, u_{1}, \sigma_{1}\right) \in D: u_{1} \in B_{m^{\prime}+N}, \sigma_{1}<L_{m^{\prime}+N}} \cdots \int_{\left(v_{m^{\prime}}, u_{m^{\prime}}, \sigma_{m^{\prime}}\right) \in D: u_{m^{\prime}} \in B_{m^{\prime}+N}, \sigma_{m^{\prime}}<L_{m^{\prime}+N}} \\
& \mathbf{1}\left[\left\{\left(v_{1}, u_{1}, \sigma_{1}\right), \ldots,\left(v_{m^{\prime}}, u_{m^{\prime}}, \sigma_{m^{\prime}}\right)\right\} \in F\right] \\
& \times h_{m^{\prime}+N}\left(v_{1}, u_{1}, \sigma_{1}\right) \ldots h_{m^{\prime}+N}\left(v_{m^{\prime}}, u_{m^{\prime}}, \sigma_{m^{\prime}}\right) \\
& \frac{\mathrm{d} \mu\left(v_{1}, u_{1}, \sigma_{1}\right)}{\mu(D)} \cdots \frac{\mathrm{d} \mu\left(v_{m^{\prime}}, u_{m^{\prime}}, \sigma_{m^{\prime}}\right)}{\mu(D)}
\end{aligned}
$$

Consequently, $P^{m^{\prime}+N}(\phi, F) \geq \varepsilon^{\prime} Q(F)$, where

$$
\begin{aligned}
\varepsilon^{\prime} & =a^{m^{\prime}+N}\left(\frac{1-q_{1}}{q_{1}}\right)^{m^{\prime}} \frac{\left[\exp \left(-L_{m^{\prime}+N} / \rho_{m^{\prime}+N}^{d}\right) \mu(D)\right]^{m^{\prime}}}{(N+1) \cdots\left(N+m^{\prime}\right)} \\
& \times \frac{m^{\prime} !}{\left(N+m^{\prime}\right) !}\left[1-\frac{\left(1-q_{1}\right) M \mu(D)}{q_{1}\left(m^{\prime}+1\right)}\right]^{N} .
\end{aligned}
$$

Notice that $Q$ is a non-zero measure, because if we set $x_{i}=x_{m}$ for $i \geq m$, then

$$
\begin{aligned}
& Q\left(\Omega_{x}\right) \geq \int_{D} \ldots \int_{D} \mathbf{1}\left[k_{u_{i}}\left(v_{i}, x_{i}\right)>0, u_{i} \in B_{m^{\prime}+N}, \sigma_{i}<L_{m^{\prime}+N}, i=1, \ldots, m^{\prime}\right] \\
& \times h_{m^{\prime}+N}\left(v_{1}, u_{1}, \sigma_{1}\right) \ldots h_{m^{\prime}+N}\left(v_{m^{\prime}}, u_{m^{\prime}}, \sigma_{m^{\prime}}\right) \frac{\mathrm{d} \mu\left(v_{1}, u_{1}, \sigma_{1}\right)}{\mu(D)} \ldots \frac{\mathrm{d} \mu\left(v_{m^{\prime}}, u_{m^{\prime}}, \sigma_{m^{\prime}}\right)}{\mu(D)}
\end{aligned}
$$


which is positive, cf. (40). Thus $S_{N}$ is a small set.

We can next verify the following geometric drift condition which implies $V$-uniform ergodicity: for each $\beta>1$ there exists constants $b<\infty$ and $b^{\prime}<1$ such that for any $\phi \in \Omega_{x}$,

$$
\mathrm{E}\left[\beta^{\operatorname{card}\left(X_{1}\right)} \mid X_{0}=\phi\right] \leq b^{\prime} \beta^{\operatorname{card}(\phi)}+b \mathbf{1}\left[\phi \in S_{N}\right]
$$

where $\left\{X_{n}\right\}_{n \geq 0}$ denotes the Metropolis-Hastings chain, and where $N>\beta M \mu(D)$. The proof follows along lines similar to those in the proof of Proposition 3.3 in [13] or Proposition 7.14 in [33] or Proposition 6 below. Finally, (44) implies $V$-uniform ergodicity, cf. Proposition 7.9 in [33].

Example 10. In applications $W_{\text {ext }}$ is bounded, and it makes not much sense to consider arbitrary large bandwidths, so let us suppose that $W_{\text {ext }}$ and $B$ are bounded, and let $\varepsilon>0$. Further, suppose that $\left\{c_{j}\right\}$ has density $\tilde{p}$ with respect to the unit rate Poisson process on $W_{\text {ext }}$, and $\left\{c_{j}\right\}$ is independent of the $\left(b_{j}, \gamma_{j}\right)$ which are i.i.d. with density $q$ (this setting covers e.g. the finite Markov point process considered in Example 4). Let the Poisson process $\nu$ be specified by the intensity measure $\mathrm{d} \mu(c, b, \gamma)=q(b, \gamma) \mathrm{d} c \mathrm{~d} b \mathrm{~d} \gamma$. Then $\Phi$ has a density with respect to $\nu$,

$$
p\left(\left\{\left(c_{1}, b_{1}, \gamma_{1}\right), \ldots,\left(c_{n}, b_{n}, \gamma_{n}\right)\right\}\right)=\tilde{p}\left(\left\{c_{1}, \ldots, c_{n}\right\}\right) .
$$

Clearly, conditions (c)-(e) are then satisfied if $k_{1}$ is positive (e.g. the Gaussian kernel (3)). If e.g. $k_{1}$ is the uniform kernel (4), then (c) and (e) still hold, and (d) is satisfied provided

$$
\int_{W_{\text {ext }}} \int_{B} \int_{\varepsilon}^{\infty} \mathbf{1}\left[\left\|c-x_{i}\right\|<b\right] q(b, \gamma) \mathrm{d} c \mathrm{~d} b \mathrm{~d} \gamma>0, \quad i=1, \ldots, m .
$$

The latter condition may be expected to hold for almost any natural choice of $W_{\text {ext }}, B, \varepsilon$, and $q$.

Suppose also that $\tilde{p}$ is a Markov density, i.e.

$$
\tilde{p}(\mathbf{c})=\prod_{y \subseteq \mathbf{c}} \varphi(y)
$$

for all finite $\mathbf{c} \subset W_{\text {ext }}$, where $\varphi$ is an interaction function, cf. (14). Then (a) is satisfied for most choices of $\varphi$ used in practice (including repulsive models and the examples considered below), cf. Chapter 6 in [33]. However, (b) is usually not satisfied. Two 
exceptions are the Strauss process [17, 43] and the area-interaction process [2, 45]: For a Strauss process,

$$
\tilde{p}\left(\left\{c_{1}, \ldots, c_{n}\right\}\right) \propto \beta^{n} \theta^{\sum_{i<j}^{1, n} \mathbf{1}\left[\left\|c_{i}-c_{j}\right\| \leq R\right]}
$$

where $\beta>0,0<\theta \leq 1$, and $R>0$ are parameters. Then (a) is satisfied with $\Lambda=\beta$, and (b) holds with $h_{K}(c, b, \gamma)=\beta \theta^{K(K+1) / 2}$. For an area-interaction process,

$$
\tilde{p}\left(\left\{c_{1}, \ldots, c_{n}\right\}\right) \propto \beta^{n} \theta^{-\left|\cup_{i=1}^{n} b\left(c_{i}, R\right)\right|}
$$

where $\beta>0, \theta>0$, and $R>0$ are parameters, and $b\left(c_{i}, R\right)$ is the ball in $\mathbb{R}^{d}$ with centre $c_{i}$ and radius $R$. Then (a) and (b) are satisfied with $\Lambda=\beta$ and $h_{K}(c, b, \gamma)=\beta \theta^{-|b(0, R)|}$ if $\theta \geq 1$, and $\Lambda=\beta \theta^{-|b(0, R)|}$ and $h_{K}(c, b, \gamma)=\beta$ if $\theta \leq 1$.

The following Proposition 6 concerns the case $q_{2}<1$ when certain conditions including the following are satisfied.

(f) $p(\phi)$ is constant and positive for all $\phi \in \Omega_{x}$ with $\operatorname{card}(\phi)=1$.

(g) $D=W_{\text {ext }} \times B \times(\varepsilon, L)$ with $W_{\text {ext }}, B, \varepsilon$ as before and where $L>\varepsilon$ is finite.

Note that the conditions in Proposition 6 imply irreducibility, and we do not need to assume (b) which, as noticed in Example 10, is violated for most Markov models. (Moreover, (d) is implied by (38) since $\mu(D)>0$, but we shall not use (d) in the proof).

Proposition 6. Assume that $q_{2}<1, \varepsilon>0$, and conditions (38), (a), (c), (e), (f), and $(g)$ are satisfied. Then for any function $V(\phi)=\beta^{\operatorname{card}(\phi)}, \phi \in \Omega_{x}$, with $\beta>1$, the birth-death-move algorithm is $V$-uniformly ergodic.

Proof. As in the proof for Proposition 5 the first step is to show that $S_{N}$ is a small set for any positive integer $N$. Let $m^{\prime}>N$ be an integer, $a=\min \left\{q_{1} q_{2},\left(1-q_{1}\right) q_{2}, 1-q_{2}\right\}$, $\phi=\left\{\left(c_{1}, b_{1}, \gamma_{1}\right),\left(c_{2}, b_{2}, \gamma_{2}\right), \ldots\left(c_{k}, b_{k}, \gamma_{k}\right)\right\} \in S_{N},\left(c_{0}^{\prime}, b_{0}^{\prime}, \gamma_{0}^{\prime}\right)=\left(c_{k}, b_{k}, \gamma_{k}\right)$, and $F \subseteq$ 
$\Omega_{x}$. Then

$$
\begin{aligned}
P^{m^{\prime}}(\phi, F) & \geq a^{m^{\prime}} \frac{1}{k !} \prod_{j=1}^{k-1} \min \left\{1, r\left[\phi \backslash\left\{\left(c_{1}, b_{1}, \gamma_{1}\right), \ldots,\left(c_{j}, b_{j}, \gamma_{j}\right)\right\},\left(c_{j}, b_{j}, \gamma_{j}\right)\right]^{-1}\right\} \\
& \times \int_{D^{m^{\prime}-k+1}} \prod_{j=1}^{m^{\prime}-k+1} \min \left\{1, \pi\left(\left\{\left(c_{j}^{\prime}, b_{j}^{\prime}, \gamma_{j}^{\prime}\right)\right\} \mid x\right) / \pi\left(\left\{\left(c_{j-1}^{\prime}, b_{j-1}^{\prime}, \gamma_{j-1}^{\prime}\right)\right\} \mid x\right)\right\} \\
& \times \mathbf{1}\left[\left\{\left(c_{m^{\prime}-k+1}^{\prime}, b_{m^{\prime}-k+1}^{\prime}, \gamma_{m^{\prime}-k+1}^{\prime}\right)\right\} \in F\right] \\
& \times \frac{\mathrm{d} \mu\left(c_{1}^{\prime}, b_{1}^{\prime}, \gamma_{1}^{\prime}\right)}{\mu(D)} \cdots \frac{\mathrm{d} \mu\left(c_{m^{\prime}-k+1}^{\prime}, b_{m^{\prime}-k+1}^{\prime}, \gamma_{m^{\prime}-k+1}^{\prime}\right)}{\mu(D)}
\end{aligned}
$$

corresponding to first deleting all except one point of $\phi$ and then applying the movement step $m^{\prime}-k+1$ times. (Note that condition (b) was used in the proof for Proposition 5 when we considered the $m^{\prime}$ additions to $\phi$; in the present proof (b) is not needed because we consider no births above). A straightforward computation shows that for any $j=1, \ldots, k-1$,

$$
\begin{aligned}
& \min \left\{1, r\left[\phi \backslash\left\{\left(c_{1}, b_{1}, \gamma_{1}\right), \ldots,\left(c_{j}, b_{j}, \gamma_{j}\right)\right\},\left(c_{j}, b_{j}, \gamma_{j}\right)\right]^{-1}\right\} \\
& \geq \min \left\{1, \frac{q_{1}}{\left(1-q_{1}\right) M \mu(D)}\right\}>0
\end{aligned}
$$

where $M>0$ is the upper bound in (39) (which was obtained without using (b)). By $(38),(\mathrm{e}),(\mathrm{f})$, and $(\mathrm{g})$, for all $(c, b, \gamma),\left(c^{\prime}, b^{\prime}, \gamma^{\prime}\right) \in D$,

$$
\begin{aligned}
\frac{\pi(\{(c, b, \gamma)\} \mid x)}{\pi\left(\left\{\left(c^{\prime}, b^{\prime}, \gamma^{\prime}\right)\right\} \mid x\right)} & =\frac{\exp \left(-\int_{W} \gamma k_{b}(c, \xi) \mathrm{d} \xi\right)}{\exp \left(-\int_{W} \gamma^{\prime} k_{b^{\prime}}\left(c^{\prime}, \xi\right) \mathrm{d} \xi\right)}\left(\frac{\gamma}{\gamma^{\prime}}\right)^{m} \prod_{j=1}^{m}\left[\frac{k_{b}\left(c, x_{j}\right)}{k_{b^{\prime}}\left(c^{\prime}, x_{j}\right)}\right] \\
& \geq \exp \left(-\gamma / b^{d}\right)\left(\frac{\gamma \delta^{\prime}}{\gamma^{\prime} \delta^{\prime \prime}}\right)^{m} \geq \exp \left(-\gamma / b^{d}\right)\left(\frac{\varepsilon \delta^{\prime}}{L \delta^{\prime \prime}}\right)^{m}
\end{aligned}
$$

where $\delta^{\prime}=\min \left\{\delta_{1}^{\prime}, \ldots, \delta_{m}^{\prime}\right\}$ and $\delta^{\prime \prime}=\max \left\{\delta_{1}^{\prime \prime}, \ldots, \delta_{m}^{\prime \prime}\right\}$. Therefore,

$$
\begin{aligned}
P^{m^{\prime}}(\phi, F) \geq \frac{a^{m^{\prime}}}{k !}[\min & \left.\left\{1, \frac{q_{1}}{\left(1-q_{1}\right) M \mu(D)}\right\}\right]^{k-1} \\
\times \int_{D} \cdots \int_{D} & \prod_{j=1}^{m^{\prime}-k+1} \min \left\{1, \exp \left(-\gamma_{j}^{\prime} / b_{j}^{\prime}\right)\left(\frac{\varepsilon \delta^{\prime}}{L \delta^{\prime \prime}}\right)^{m}\right\} \\
& \times \mathbf{1}\left[\left\{\left(c_{m^{\prime}-k+1}^{\prime}, b_{m^{\prime}-k+1}^{\prime}, \gamma_{m^{\prime}-k+1}^{\prime}\right)\right\} \in F\right] \\
& \frac{\mathrm{d} \mu\left(c_{1}^{\prime}, b_{1}^{\prime}, \gamma_{1}^{\prime}\right)}{\mu(D)} \cdots \frac{\mathrm{d} \mu\left(c_{m^{\prime}-k+1}^{\prime}, b_{m^{\prime}-k+1}^{\prime}, \gamma_{m^{\prime}-k+1}^{\prime}\right)}{\mu(D)} .
\end{aligned}
$$

Since $\mu(D)>0$, there exists $B^{\prime} \subseteq B$ such that $\rho \equiv \inf B^{\prime} \in(0, \infty)$ and $\mu\left(W_{\text {ext }} \times B^{\prime} \times\right.$ 
$(\epsilon, L))>0$. Thus

$$
\begin{aligned}
P^{m^{\prime}}(\phi, F) \geq & \frac{a^{m^{\prime}}}{k !}\left[\min \left\{1, \frac{q_{1}}{\left(1-q_{1}\right) M \mu(D)}\right\}\right]^{k-1} \\
\times & \int_{D} \ldots \int_{D} \prod_{j=1}^{m^{\prime}-k+1} \min \left\{1, \exp \left(-\gamma_{j}^{\prime} / b_{j}^{\prime}\right)\left(\frac{\varepsilon \delta^{\prime}}{L \delta^{\prime \prime}}\right)^{m}\right\} \\
& \times \mathbf{1}\left[b_{1}^{\prime} \in B^{\prime}, \ldots, b_{m^{\prime}-k+1}^{\prime} \in B^{\prime},\left\{\left(c_{m^{\prime}-k+1}^{\prime}, b_{m^{\prime}-k+1}^{\prime}, \gamma_{m^{\prime}-k+1}^{\prime}\right)\right\} \in F\right] \\
& \quad \frac{\mathrm{d} \mu\left(c_{1}^{\prime}, b_{1}^{\prime}, \gamma_{1}^{\prime}\right)}{\mu(D)} \cdots \frac{\mathrm{d} \mu\left(c_{m^{\prime}-k+1}^{\prime}, b_{m^{\prime}-k+1}^{\prime}, \gamma_{m^{\prime}-k+1}^{\prime}\right)}{\mu(D)} \\
\geq & \left.\frac{a^{m^{\prime}}}{k !}\left[\min \left\{1, \frac{q_{1}}{\left(1-q_{1}\right) M \mu(D)}\right\}\right]^{k-1}\right]^{m^{m^{\prime}-k+1}}\left(\frac{\mu\left(D^{\prime}\right)}{\mu(D)}\right)^{m^{\prime}-k} Q(F) \\
\times & {\left[\min \left\{1, \exp \left(-L / \rho_{j}^{d}\right)\left(\frac{\varepsilon \delta^{\prime}}{L \delta^{\prime \prime}}\right)^{m}\right\}\right]^{m^{\prime}} }
\end{aligned}
$$

where $D^{\prime}=W_{\text {ext }} \times B^{\prime} \times(\varepsilon, L)$ and

$$
Q(F)=\int_{D^{\prime}} \mathbf{1}[\{(v, u, \sigma)\} \in F] \frac{\mathrm{d} \mu(v, u, \sigma)}{\mu(D)} .
$$

Notice that $Q$ is a non-zero measure, since by (38) and (f), $Q\left(\Omega_{x}\right)=\mu\left(D^{\prime}\right) / \mu(D)>0$.

Furthermore, $P^{m^{\prime}}(\phi, F) \geq \varepsilon^{\prime} Q(F)$ where

$$
\begin{aligned}
\varepsilon^{\prime} & =\frac{a^{m^{\prime}}}{N !}\left[\min \left\{1, \frac{q_{1}}{\left(1-q_{1}\right) M \mu(D)}\right\}\right]^{N-1} \\
& \times\left[\min \left\{1, \exp \left(-L / \rho^{d}\right)\left(\frac{\varepsilon \delta^{\prime}}{L \delta^{\prime \prime}}\right)^{m}\right\}\right]^{m^{\prime}+1}\left(\frac{\mu\left(D^{\prime}\right)}{\mu(D)}\right)^{m^{\prime}}>0 .
\end{aligned}
$$

Thus $S_{N}$ is a small set.

Next we establish $V$-uniform ergodicity by checking the geometric drift condition (44) with $N>\max \left\{\beta,\left(1-q_{1}\right) / q_{1}\right\} M \mu(D)$. Assume that the current state $\phi$ of the Metropolis-Hastings chain is such that $\phi \notin S_{N}$, and set $k=\operatorname{card}(\phi)$. As for the birthdeath Metropolis-Hastings algorithm, here again the probability of accepting a death is 1 . Now, letting

$$
B(\phi)=\int_{D} \min \{1, r[\phi,(c, b, \gamma)]\} \frac{d \mu(c, b, \gamma)}{\mu(D)}
$$

denote the probability of accepting a birth, we have that

$$
B(\phi) \leq \frac{\left(1-q_{1}\right) M \mu(D)}{q_{1}(k+1)} \leq \frac{\left(1-q_{1}\right) M \mu(D)}{q_{1}(N+1)},
$$


since $\phi \notin S_{N}$. So

$$
\begin{aligned}
& \mathrm{E}\left[\beta^{\operatorname{card}\left(X_{1}\right)} \mid X_{0}=\phi\right] \\
= & q_{1} q_{2} \beta^{k+1} B(\phi)+q_{1} q_{2} \beta^{k}(1-B(\phi))+\left(1-q_{1}\right) q_{2} \beta^{k-1}+\left(1-q_{2}\right) \beta^{k} \\
\leq & \beta^{k}\left[\left(1-q_{1}\right) q_{2}(\beta-1) \frac{M \mu(D)}{N+1}+\left(1-q_{1}\right) q_{2}\left(\frac{1}{\beta}-1\right)+1\right]
\end{aligned}
$$

where we have used that $\beta>1$. Therefore, when $\phi \notin S_{N}$, (44) follows setting $b^{\prime}$ equal to $[\cdots]$ from (45). Indeed, since $N>\max \left\{\beta,\left(1-q_{1}\right) / q_{1}\right\} M \mu(D)$, it is easily seen that $b^{\prime}<1$. Finally, when $\phi \in S_{N}$,

$$
\mathrm{E}\left[\beta^{\operatorname{card}\left(X_{1}\right)} \mid X_{0}=\phi\right] \leq \beta^{\operatorname{card}(\phi)+1} \leq \beta^{N+1}
$$

whereby (44) is verified.

Example 11. Conditions (38) and (c) are satisfied if $k_{1}$ is positive, but as mentioned (38) may easily be violated for the uniform kernel. If the situation is as in Example 10, then (f) means that $\varphi(y)$ is a constant $\beta>0$ whenever $\operatorname{card}(y)=1$. If this is not the case, (a) can still be obtained by redefining the intensity measure of $\nu$ as $\mathrm{d} \mu(c, b, \gamma)=$ $\varphi(\{c\}) q(b, \gamma) \mathrm{d} c \mathrm{~d} b \mathrm{~d} \gamma$. Finally, (g) is needed for technical reasons, cf. the proof above, and the upper bound $L$ on the $\gamma_{j}$ may in many applications be a less serious assumption.

\section{Concluding remarks}

Although we have demonstrated that GSNCPs to some extend possess many appealing properties, it remains to investigate the statistical aspects and practical use of GSNCPs. The results in Section 3.1 for the intensity and pair correlation function may be useful for model checking and parameter estimation, particularly minimum contrast estimation, see [33]. The results in Section 3.2 on reduced Palm distributions and $J$-functions may to some extend be useful for statistical applications [20, 21, 33]. The most important part of this paper from a statistical viewpoint is probably the simulation algorithms in Section 4, since they provide ways of performing simulationbased inference, cf. Section 2.1 and [33]. The algorithms in Sections 4.1 and 4.2 for simulation of GSNCPs may be useful in connection to model checking, while the conditional simulation algorithm in Sections 4.3 will be needed in connection to both Bayesian and likelihood inference, cf. [33]. 
In this paper we have concentrated on the probabilistic aspects of GSNCPs. Future research should address the following: 1) Markov properties of GSNCPs, cf. the discussion in [29] for SNCPs. 2) Implementation and experimentation with the simulation algorithms treated in Section 4. 3) Convergence properties of MCMC hybrid (or Gibbs within Metropolis, see e.g. [10]) algorithms for conditional simulation, when we extend the setting in Section 4.3 by imposing a prior distribution on hyperparameters for the distribution of $\Phi$. 4) Exploit our current understanding of GSNCPs for related models, e.g. spatial Hawkes processes (see point (e) in Section 2.1).

\section{Acknowledgements}

This research was supported by the Danish Natural Science Research Council, the Network in Mathematical Physics and Stochastics (MaPhySto; funded by a grant from the Danish National Research Foundation), and the Murst Project "Processi Stocastici e applicazioni a Filtraggio, Controllo, Simulazione e Finanza Matematica".

\section{References}

[1] Baddeley, A. ANd Møller, J. (1989). Nearest-neighbour Markov point processes and random sets. International Statistical Review 2, 89-121.

[2] Baddeley, A. J. and van Lieshout, M. N. M. (1995). Area-interaction point processes. Annals of the Institute of Statistical Mathematics 46, 601-619.

[3] Brix, A. (1999). Generalized gamma measures and shot-noise Cox processes. Advances in Applied Probability 31, 929-953.

[4] Brix, A. and Chadoeuf, J. (2000). Spatio-temporal modeling of weeds and shot-noise G Cox processes. Submitted.

[5] Brix, A. And Diggle, P. J. (2001). Spatio-temporal prediction for log-Gaussian Cox processes. Journal of the Royal Statistical Society Series B 63, 823-841.

[6] Brix, A. and Kendall, W. S. (2002). Simulation of cluster point processes without edge effects. Advances in Applied Probability 34, 267-280. 
[7] Brix, A. And Møller, J. (2001). Space-time multitype log Gaussian Cox processes with a view to modelling weed data. Scandinavian Journal of Statistics 28, 471-488.

[8] Cox, D. R. And Isham, V. (1980). Point Processes. Chapman \& Hall, London.

[9] Daley, D. J. And Vere-Jones, D. (2003). An Introduction to the Theory of Point Processes. Volume I: Elementary Theory and Methods second ed. SpringerVerlag, New York.

[10] Dellaportas, P. and Roberts, G. O. (2003). An introduction to MCMC. In Spatial Statistics and Computational Methods. ed. J. Møller. Lecture Notes in Statistics 173. Springer-Verlag, New York pp. 1-41.

[11] Diggle, P. J. (2003). Statistical Analysis of Spatial Point Patterns (second edition). Arnold, London.

[12] Georgit, H.-O. (1976). Canonical and grand canonical Gibbs states for continuum systems. Communications of Mathematical Physics 48, 31-51.

[13] Geyer, C. J. (1999). Likelihood inference for spatial point processes. In Stochastic Geometry: Likelihood and Computation. ed. O. E. Barndorff-Nielsen, W. S. Kendall, and M. N. M. van Lieshout. Chapman \& Hall/CRC, Boca Raton, Florida. pp. $79-140$.

[14] Geyer, C. J. AND Møller, J. (1994). Simulation procedures and likelihood inference for spatial point processes. Scandinavian Journal of Statistics 21, 359373.

[15] Hawkes, A. G. (1971). Spectra of some self-exciting and mutually exciting point processes. Biometrika 58, 83-90.

[16] Hawkes, A. G. And OAKes, D. (1974). A cluster representation of a self-exciting process. Journal of Applied Probability 11, 493-503.

[17] Kelly, F. P. And Ripley, B. D. (1976). A note on Strauss' model for clustering. Biometrika 63, 357-360. 
[18] Kendall, W. S. and Møller, J. (2000). Perfect simulation using dominating processes on ordered spaces, with application to locally stable point processes. Advances in Applied Probability 32, 844-865.

[19] Kingman, J. F. C. (1977). Remarks on the spatial distribution of a reproducing population. Journal of Applied Probability 14, 577-583.

[20] Lieshout, M. N. M. van (2000). Markov Point Processes and Their Applications. Imperial College Press, London.

[21] Lieshout, M. N. M. van and Baddeley, A. J. (1996). A nonparametric measure of spatial interaction in point patterns. Statistica Neerlandica 50, 344361.

[22] Lieshout, M. N. M. van and Baddeley, A. J. (2002). Extrapolating and interpolating spatial patterns. In Spatial Cluster Modelling. ed. A. B. Lawson and D. Denison. Chapman \& Hall/CRC, Boca Raton, Florida pp. 61-86.

[23] Lindvall, T. (1992). Lectures on the Coupling Method. Wiley, NewYork.

[24] Matérn, B. (1960). Spatial Variation, Meddelanden från Statens Skogforskningsinstitut, 49 (5).

[25] Matérn, B. (1986). Spatial Variation. Lecture Notes in Statistics 36, SpringerVerlag, Berlin.

[26] Meyn, S. P. And Tweedie, R. L. (1993). Markov Chains and Stochastic Stability. Springer-Verlag, London.

[27] Møller, J. (1999). Markov chain Monte Carlo and spatial point processes. In Stochastic Geometry: Likelihood and Computation. ed. O. E. Barndorff-Nielsen, W. S. Kendall, and M. N. M. van Lieshout. Monographs on Statistics and Applied Probability 80, Chapman \& Hall/CRC, Boca Raton, Florida pp. 141-172.

[28] MøLLER, J. (2003). A comparison of spatial point process models in epidemiological applications. In Highly Structured Stochastic Systems. ed. P. J. Green, N. L. Hjort, and S. Richardson. Oxford University Press, Oxford pp. 264-268. 
[29] Møller, J. (2003). Shot noise Cox processes. Advances in Applied Probability 35, 614-640.

[30] Møller, J., Syversveen, A. R. And Waagepetersen, R. P. (1998). Log Gaussian Cox processes. Scandinavian Journal of Statistics 25, 451-482.

[31] Møller, J. And Waagepetersen, R. P. (2002). Statistical inference for Cox processes. In Spatial Cluster Modelling. ed. A. B. Lawson and D. Denison. Chapman \& Hall/CRC, Boca Raton, Florida pp. 37-60.

[32] Møller, J. and Waagepetersen, R. P. (2003). An introduction to simulationbased inference for spatial point processes. In Spatial Statistics and Computational Methods. ed. J. Møller. Lecture Notes in Statistics 173. Springer-Verlag, New York pp. $143-198$.

[33] Møller, J. And WaAgepetersen, R. P. (2003). Statistical Inference and Simulation for Spatial Point Processes. Chapman and Hall/CRC, Boca Raton.

[34] Müller, A. And Stoyan, D. (2002). Comparison Methods for Stochastic Models and Risks. Wiley, Chichester.

[35] Neyman, J. And Scott, E. L. (1958). Statistical approach to problems of cosmology. Journal of the Royal Statistical Society Series B 20, 1-43.

[36] Nguyen, X. X. And Zessin, H. (1979). Integral and differential characterizations of Gibbs processes. Mathematische Nachrichten 88, 105-115.

[37] Preston, C. (1976). Random Fields. Lecture Notes in Mathematics 534. Springer-Verlag, Berlin.

[38] Richardson, S. (2003). Spatial models in epidemiological applications. In Highly Structured Stochastic Systems. ed. P. J. Green, N. L. Hjort, and S. Richardson. Oxford University Press, Oxford pp. 237-259.

[39] Ripley, B. D. (1977). Modelling spatial patterns (with discussion). Journal of the Royal Statistical Society Series B 39, 172-212.

[40] Ripley, B. D. And Kelly, F. P. (1977). Markov point processes. Journal of the London Mathematical Society 15, 188-192. 
[41] Ruelle, D. (1969). Statistical Mechanics: Rigorous Results. W.A. Benjamin, Reading, Massachusetts.

[42] Stoyan, D., Kendall, W. S. And Mecke, J. (1995). Stochastic Geometry and Its Applications second ed. Wiley, Chichester.

[43] Strauss, D. J. (1975). A model for clustering. Biometrika 63, 467-475.

[44] Thomas, M. (1949). A generalization of Poisson's binomial limit for use in ecology. Biometrika 36, 18-25.

[45] Widom, B. And Rowlinson, J. S. (1970). A new model for the study of liquidvapor phase transitions. Journal of Chemical Physics 52, 1670-1684.

[46] Wolpert, R. L. And Ickstadt, K. (1998). Poisson/gamma random field models for spatial statistics. Biometrika 85, 251-267. 